OPEN ACCESS

Edited by: Nigel Turner,

University of New South Wales,

Australia

Reviewed by:

Andrea Armani,

San Raffaele Pisana (IRCCS), Italy

Simon Timothy Bond

Baker Heart and Diabetes Institute,

Australia

*Correspondence:

Sadia Kanwal

sadiakanwa/490@gmail.com

Specialty section:

This article was submitted to

Obesity,

a section of the journal

Frontiers in Endocrinology

Received: 04 May 2020

Accepted: 13 October 2020

Published: 19 November 2020

Citation:

Kanwal S, Aliya S and Xin Y (2020)

Anti-Obesity Effect of Dictyophora indusiata Mushroom Polysaccharide (DIP) in High Fat Diet-Induced Obesity via Regulating Inflammatory Cascades and Intestinal Microbiome.

Front. Endocrinol. 11:558874. doi: 10.3389/fendo.2020.558874

\section{Anti-Obesity Effect of Dictyophora indusiata Mushroom Polysaccharide (DIP) in High Fat Diet-Induced Obesity via Regulating Inflammatory Cascades and Intestinal Microbiome}

\author{
Sadia Kanwal ${ }^{1 *}$, Shams Aliya ${ }^{2}$ and Yi Xin ${ }^{1}$ \\ ${ }^{1}$ Department of Biotechnology, College of Basic Medical Sciences, Dalian Medical University, Dalian, China, ${ }^{2}$ Faculty of Life \\ Sciences, Institute of Cancer Therapeutics, University of Bradford, Bradford, United Kingdom
}

Obesity is a multifactorial metabolic disorder characterized by low-grade chronic inflammation, hyper-permeability of the gut epithelium, and perturbation of the intestinal microbiome. Despite the numerous therapeutic efficacies of Dictyophora indusiata mushroom, its biological activity in alleviating obesity through regulation of the gut microbiota and inflammatory cascades remain obscure. Henceforth, we determined the modulatory impact of $D$. indusiata polysaccharide (DIP) in the high-fat diet (HFD)-induced obesity mice model. The experimental subjects (BALB/C mice) were supplemented with chow diet (Control group), high-fat diet (HFD group), or HFD along with DIP at a low dose $[H F D+D I P(L)]$ and high dose $[H F D+D I P(H)]$. Obesity-related parameters, including body weight gain, epididymal adipocyte size, fat accumulation, adipogenic markers, lipogenic markers, inflammatory associated markers, intestinal integrity, and intestinal microbiome, were elucidated. Our findings demonstrated that the oral administration of DIP at low dose partially and at high dose significantly reversed HFD-induced obesity parameters. Furthermore, the body weight, fat accumulation, adipocyte size, adipogenic and liver associated markers, glucose levels, inflammatory cytokines, and endotoxin (Lipopolysaccharide, LPS) levels were reduced considerably. Moreover, the study revealed that DIP treatment reversed the dynamic alterations of the gut microbiome community by decreasing the Firmicutes to Bacteroidetes ratio. These findings led us to infer the therapeutic potential of DIP in alleviating HFD-induced obesity via regulating inflammatory cascades, modulating intestinal integrity and intestinal microbiome community.

Keywords: obesity, high-fat diet, gut microbiota, inflammation, Dictyophora indusiata polysaccharide (DIP) 


\section{INTRODUCTION}

Obesity is one of the major health concerns across the world that has been implicated with multiple health problems and a reduced life expectancy $(1,2)$. The prevalence of obesity has been strikingly increased in recent years, and it has been estimated that by 2030 obesity rate will reach up to $20 \%$ of the adult population (3). Approximately 500 million individuals are obese, and 1.4 billion are overweight globally (4). Obesity is characterized by excessive fat accumulation due to various factors, including genetic predisposition, high-calorie energy intake, and sedentary lifestyle (5). Obesity predisposes an individual to develop and aggravate a broad spectrum of disorders, including type 2 diabetes, insulin resistance, fatty liver disease, gastrointestinal problems, cardiovascular disease, respiratory problems, and various kinds of cancers (6-9). Considering the adverse impact of obesity on individual health, it is of great challenge to reduce and halt obesity in the modern world.

A growing body of evidence demonstrates that a common trait identified in obese humans and animal studies is the hyper permeable gut $(10,11)$. A leaky gut eventually causes local or systemic inflammation due to increase susceptibility to antigens leading to impairment of gut barrier function, reduced tight junction protein (TJP) expression, and increased intestinal permeability to pathogenic microorganism (12-15). Moreover, high fat-induced obesity enhances lipopolysaccharide (LPS) levels in the bloodstream, ultimately causing inflammation and insulin resistance $(13,16,17)$. LPS is the main component in synthesizing the outer membrane of gram-negative bacteria that triggers several transcription factors associated with inflammation resulting in intestinal barrier damage (18). Furthermore, many reports have shown enhanced pro-inflammatory cytokine production, such as tumor necrosis factor- $\alpha$ (TNF- $\alpha$ ), interleukin (IL)-1 $\beta$, IL-6, and reduced anti-inflammatory cytokine secretion in obese animal models $(19,20)$. Another study shows that a higher level of cytokines triggers not only chronic inflammation but also insulin resistance (7).

The role of the gut microbiota in the predisposition of obesity and related disorders is well recognized (21-23). The intestinal flora maintains the host physiology via harnessing energy from complex and indigestible dietary compounds such as polysaccharides and polyphenols (24-27). The alteration of bacterial composition leads to a condition known as gut dysbiosis (28-30). Gut microbiome perturbation (dysbiosis) has been implicated with several human ailments, including inflammatory diseases, obesity, metabolic, and neurological disorders (31-35). High fat dietary intake is associated with the alteration of the composition of the intestinal microbiota community, distinguished by reduced bacterial diversity and richness, and increase abundance of Firmicutes to Bacteroidetes ratio $(15,36-41)$. Various in-vivo studies have demonstrated that gut dysbiosis is a major contributing factor in obesity and type 2 diabetes (42-44). Furthermore, clinical studies have also proven the association of intestinal dysbiosis in the etiology of obesity and obesity-related disorders (45). Thus the dynamic shift of intestinal flora due to high-fat diet consumption not only develops obesity but also disrupts intestinal integrity and increases endotoxemia (LPS) levels (16). The enhanced LPS levels eventually trigger inflammatory cascades and enhance insulin resistance due to the activation of inflammatory signaling pathways $(13,17,46,47)$. Nevertheless, the modulation of gut microbiome through dietary interventions, including herbal plants, probiotics/ prebiotics, is promising strategies to reduce inflammatory-related problems such as obesity and associated metabolic syndrome (48-53).

Several drugs have been developed in recent years to combat obesity and obesity-related health issues. Nonetheless, long term dependency on anti-obesity drugs has been implicated with severe side effects and weight regain upon discontinuation (54-56). Henceforth researchers are focusing on finding novel therapeutic arsenals to prevent this social and economic catastrophe and overcome obesity-associated problems. In this regard, the role of natural dietary compounds such as mushroom polysaccharides in treating multiple diseases is drastically increasing, owing to the plethora of health ameliorating properties. The prebiotic sources such as mushroom polysaccharides that serve as a substrate for microbes residing in the gastrointestinal tract enhance the growth of microbes that exert a beneficial physiological impact on host immunity (57-60). Additionally, dietary fiber encourages the growth of short-chain fatty acids' (SCFAs') producing bacteria that inhibit inflammatory reactions $(15,58,61,62)$. Moreover, prebiotics not only exerts a beneficial impact on the gut microbial community but also modulates gut barrier integrity via regulating TJP expression and reducing endotoxin levels in the bloodstream (63).

Accumulated pieces of evidence suggest that Dictyophora indusiata mushroom derived polysaccharides possess a variety of biological activities (64) such as anti-inflammatory, antitumor, antioxidant, and immunomodulatory activities (65-68). In our previous studies, we have reported the modulatory effect of Dictyophora indusiata polysaccharide (DIP) in antibiotic driven intestinal dysbiosis and DSS induced ulcerative colitis $(69,70)$. The anti-obesity effect of DIP via regulation of obesityassociated parameters and improving gut microbiome community is yet to be explored. Given the protective effect of Dictyophora indusiata mushroom in combating various health problems, in the present study, we further investigated the effect of DIP on HFD-induced obesity. Our findings revealed that HFD-induced obesity was reduced upon DIP supplementation. The intestinal integrity and inflammatory reactions were ameliorated. Moreover, the gut microbiome shift was reversed. These results thus demonstrate that the D. indusiata derived polysaccharide (DIP) may be used as a potential prebiotic compound in treating obesity and associated issues.

\section{MATERIALS AND METHODS}

\section{Experimental Subjects and Housing}

Specific-pathogen-free (SPF) level inbred male BALB/c mice aged seven weeks old with similar initial body weight $(20 \pm$ 3 g) approved by the Animal Care and Research Ethics Committee of Dalian Medical University, China (Approval Number: SYXK 2016-2018) were randomly assigned to four 
groups (eight mice per group). All the animals used in the study were acclimated for a period of one week prior to experimentation. All experimental procedures were approved by the ethics committee of Dalian Medical University. The experimental subjects of the respective group were housed randomly in two separate cages (four mice per cage, so two separate cages for each group). All the cages were maintained in an environmentally controlled room $\left(20-22^{\circ} \mathrm{C}\right)$ temperature and $55 \pm 5 \%$ relative humidity with $12-12 \mathrm{~h} \mathrm{light/dark}$ cycle). The experimental design is presented in Figure 1. The mice were placed on standard commercial chow diet (control group) or $60 \%$ high-fat diet (HFD groups) (Jiangsu Medison Biomedical Co., Ltd., Yangzhou, Jiangsu Province, China) for 8 weeks. On $4^{\text {th }}$ week, the chow and HFD group were gavaged with water (200 $\mu \mathrm{l})$. The crude polysaccharide (DIP) from $D$. indusiata was administered $(200 \mu \mathrm{l})$ daily at a low dose [HFD + DIP(L) group] of $200 \mathrm{mg} / \mathrm{kg}$ body weight and a high dose [HFD+DIP (H) group] of $400 \mathrm{mg} / \mathrm{kg}$ body weight via oral gavage from week $4^{\text {th }}$ till the $8^{\text {th }}$ week. The dosage and treatment duration are based upon a previous study (71). The bodyweight of each subject from the respective group was monitored once a week, and food intake was measured every day in the morning. After 8 weeks, all the experimental subjects were sacrificed. The organs, tissues, and blood samples were collected for the subsequent analysis.

\section{Antibodies, Kits, and Reagents}

The fruiting body of the mushroom $D$. indusiata was obtained from Anhui Joy Lok Food Co., Ltd., Ningde, Fujian Province, China. The DNA extraction kit (QIAamp DNA Stool Mini Kit) and gel purification kit (Agencourt AMPure XP $60 \mathrm{~mL}$ Kit) were from Qiagen (Hilden, Germany) and Beckman Coulter (Brea, CA, USA), respectively. The primary antibodies $\beta$-actin, claudin- 1 , occludin, zonula occludens-1 (ZO-1), secondary antibodies, and the
Radioimmunoprecipitation assay (RIPA) buffer were from Proteintech (Wuhan, China). The horseradish peroxidaseconjugated secondary antibody was from ZSGB-BIO (Beijing, China). The bicinchoninic acid (BCA) protein assay kit was from Pierce Rockford, IL, USA. Polyvinylidene difluoride (PVDF) membranes were from Immobilon TM-P, Millipore, Massachusetts, USA, and WesternBright ${ }^{\mathrm{TM}}$ ECL substrate was from Advansta, Inc., Menlo Park, CA, USA. The ELISA kits were purchased from Shang Hai Lengton Bioscience Co, Ltd., Shanghai, China. All the other chemicals used in this study were of analytical grade and purchased from standard commercial sources.

\section{Extraction and Chemical Analysis of Crude Polysaccharide DIP From the Mushroom D. indusiata}

The polysaccharide extraction method, chemical analysis, and monosaccharide composition are described in our previous study (69). Briefly, the sugar content and crude polysaccharide were determined with bicinchoninic acid (BCA) and phenol sulfuric acid method. The monosaccharide composition was determined with high-performance liquid chromatography (HPLC). The polysaccharide yield was $13.2 \%$, and the main functional components were glucose $59.84 \%$, mannose $23.55 \%$, and galactose $12.95 \%$.

\section{Morphometry Analysis of Adipose Tissues}

Histological analysis was performed to determine the size of adipose tissue. Freshly isolated epididymal adipose tissues from all the experimental groups were fixed overnight in $10 \%$ formalin. The tissue sections were deparaffinized and embedded in paraffin, followed by sectioning. $5 \mu \mathrm{m}$ paraffin sections were stained with hematoxylin and eosin, and images were obtained using a microscope (Leica Microsystems, Wetzlar,

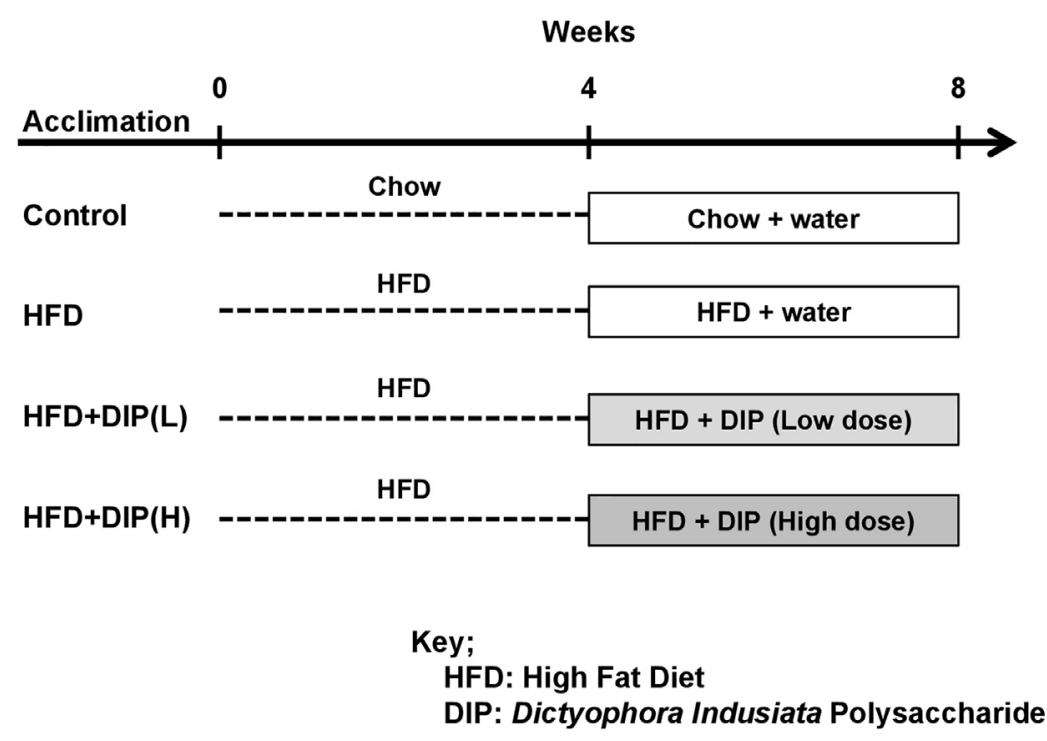

FIGURE 1 | Experimental design. Experimental mice were categorized into four groups ( $\mathrm{n}=8$ each group). Control or vehicle group, HFD group, HFD followed by $\mathrm{DIP}(\mathrm{L})$ group and HFD followed by $\mathrm{DIP}(\mathrm{H})$ group. 
Germany). For the measurement of adipocytes, the number of adipocytes was counted for each sample from the respective group, and the average adipocyte size was analyzed using the Image J software (National Institutes of Health, USA) as described previously (72).

\section{Determination of Blood Insulin and Glucose}

Blood insulin levels of overnight fasted ( $12 \mathrm{~h}$ ) mice were measured using a commercial ELISA kit (Wuhan Cusabio Biotech Co., Ltd., China) according to the manufacturer's recommendations. Blood glucose of overnight fasted $(12 \mathrm{~h})$ mice was determined with a glucose meter (Roche Diagnostics, Switzerland).

\section{Biochemical Analysis and Cytokine Level Measurement}

Blood samples were centrifuged at $3,000 \times \mathrm{g}$ for $10 \mathrm{~min}$, and serum was separated to evaluate serum endotoxin (lipopolysaccharide, LPS) levels using a commercial kit based on the guidelines provided by the supplier (LAL, Limulus amebocyte lysate assay; Chinese Horseshoe Crab Reagent Manufactory Co., Ltd. Xiamen, China). The pro-inflammatory cytokine levels including tumor necrosis factor-alpha (TNF- $\alpha$ ), interleukin 6 (IL-6), interleukin $1 \beta$ (IL-1 $\beta$ ), and anti-inflammatory cytokines such as interleukin 4 (IL-4) and interleukin 10 (IL-10) in the serum were determined using a mouse ELISA kit (Shang Hai Lengton Bioscience Co., Ltd.) Shanghai, as per manufacturer's instructions. Alanine transaminase (ALT), aspartate transaminase (AST), total cholesterol (TC), and triglyceride (TG) levels were estimated using a chemical analyzer (Hitachi, Tokyo, Japan). Serum free fatty acids (FFAs) were measured using a commercial kit (Biovision, USA).

\section{RNA Extraction and Quantitative Real- Time Reverse-Transcription PCR}

The RNA was extracted using TRIzol reagent (Invitrogen Life Technology Gaithersburg, MD, USA) according to the manufacturers' instructions. Quantitative real-time reversetranscription PCR (qRT-PCR) was performed in triplicate using SYBR Green (Kapa SYBR Fast Master Mix) and RealTime PCR thermocycler (Applied Biosystems StepOnePlus ${ }^{\mathrm{TM}}$ ). The total volume of the reaction mixture for each tube was $10 \mu \mathrm{l}$ containing $1.5 \mu \mathrm{l}$ of cDNA, $1 \mu \mathrm{l}$ of target primers, $2.5 \mu \mathrm{l}$ of nuclease-free water, and $5 \mu$ l of SYBR Green Master Mix. RTPCR was performed for 50 cycles, and PCR conditions were as follows: Initial pre-incubation at $95^{\circ} \mathrm{C}$ for $10 \mathrm{~min}$, denaturation for $15 \mathrm{~s}$ at $94^{\circ} \mathrm{C}$, annealing for $30 \mathrm{~s}$ at $60^{\circ} \mathrm{C}$ followed by elongation for $30 \mathrm{~s}$ at $72^{\circ} \mathrm{C}$. The relative gene expression was quantified by using the $2^{-\Delta \Delta C t}$ method (73). GAPDH was used as an internal control, and all the reactions were performed in triplicate. The primer sequences used in the study are shown in Supplementary Table S1.

\section{Immunoblotting Assay}

Intestinal tissue samples (100 mg) were homogenized using RIPA lysis buffer containing a protease inhibitor (Transgene Biotech, Beijing, China) and then centrifuged at $12,000 \mathrm{~g}$ for $5 \mathrm{~min}$ at $4^{\circ} \mathrm{C}$. The supernatant containing protein was removed from the pellet, and the protein concentration was determined using the BCA protein assay kit (Pierce, Rockford, IL, USA) according to the manufacturer's instructions. $10 \%$ sodium dodecyl sulfatepolyacrylamide gel (SDS PAGE) was employed for fractionation of protein lysates followed by blotting onto Polyvinylidene difluoride (PVDF) membrane. 5\% non-fat milk in TBST was used for blocking non-specific binding for $1 \mathrm{~h}$ at room temperature. After blocking, membranes were probed overnight at $4^{\circ} \mathrm{C}$ with primary antibodies including $\beta$-actin (catalog no. 20536-1-AP, 1:2000), claudin-1 (catalog no. 13050-1-AP, 1:500), occludin (catalog no. 13409-1AP, 1:2000), and ZO-1 (catalog no. 21773-1-AP, 1:500). Membranes were then incubated with horseradish peroxidase-conjugated secondary antibody (IgG) (catalog no. SA00001-2, 1:6,000) for $1 \mathrm{~h}$ at room temperature. Protein bands were developed with WesternBright ${ }^{\text {TM }}$ ECL substrate (Advansta, Inc., Menlo Park, CA, USA), and images were captured by ChemoDocTM XRS + ImagerBio-Rad (Bio-Rad Laboratories, Inc., Hercules, CA, USA).

\section{DNA Extraction and 16S rRNA Gene Amplification (Illumina MiSeq Sequencing)}

Metagenomic DNA extraction, 16S rRNA gene amplification, and sequencing were executed as described in our previous study (69). Briefly, the 16S rRNA gene V4 region was amplified from genomic DNA using the universal bacterial primers: $\left(515 \mathrm{~F}, 5^{\prime}\right.$ GTGCCAGCMGCCGCGGTAA - 3' and 806R, 5' GGACTACHVGGGTWTCTAAT-3'). The amplicons were normalized, pooled, and sequenced on the Illumina Hiseq4000 sequencer $(2 \times 150$ bp paired-end $)$. Libraries were prepared using Library Quant Kit Illumina GA revised primer-SYBR Fast Universal (KAPA, Wilmington, MA, USA) and sequenced for 600 cycles on an Illumina MiSeq using the MiSeq Reagent Kit (Illumina, San Diego, CA, USA). For bioinformatics analysis, high-quality reads were selected, and effective reads were clustered into OTUs based on $97 \%$ sequence similarity. Sequence read processing was performed using Quantitative Insights into Microbial Ecology (QIIME) (version 1.9.0). Low-quality reads that did not align to the reference alignment were removed. Chimeras identified using Chimera.UCHIME were also eliminated. Taxonomic assignment of individual datasets was performed using the SILVA128. Operational taxonomic units (OTUs) were generated using Vsearch v1.11.1 with a dissimilarity cutoff of 0.03 . Bacterial diversity within the community, such as the relative abundance of bacterial flora, species richness, and evenness, was determined using alpha diversity ( $\alpha$-diversity) indices, including Shannon index, observed species, and rank abundance curve with QIIME. To demonstrate variation among different treatment groups, beta-diversity ( $\beta$-diversity) indices were determined, including cluster dendrogram and principal coordinate analysis (PCoA) using QIIME.

\section{Statistical Analysis}

All the statistical analysis was performed with GraphPad Prism 7.04 software (La Jolla, CA, USA). The normal distribution for parametric data is elucidated with the D'Agostino \& Pearson normality test. Statistical significance was determined by using analysis of variance (ANOVA) one-way and two-way analysis of variance followed by Tukey's and Bonferroni's multiple comparison test, and $\mathrm{p}<0.05$ was considered to be statistically significant. 


\section{RESULTS}

\section{DIP Intervention Reduced HFD-Induced Obesity-Related Parameters in BALB/C Mice}

To study the effect of DIP on obesity-related parameters, we used BALB/C mice model and fed mice with HFD for 8 weeks. HFD feeding for 8 weeks led to a significant increment of the body weight, liver weight, epididymal, and subcutaneous fat accumulation compared with the control group (Figures 2A-F). Our findings revealed a significant reduction of body weight, liver weight, epididymal, and subcutaneous fat accumulation in DIP intervention groups in a dose-dependent manner as compared to the HFD alone group (Figures 2A-F). The mean body weight was significantly higher in the HFD group $(43.5 \pm 3 \mathrm{~g})$ as compared to $\mathrm{HFD}+\mathrm{DIP}(\mathrm{L})(41 \pm 3 \mathrm{~g})$ and HFD + DIP(H) (38 \pm 3 g) groups respectively (Figure 2A). The statistically significant weight gain reduction was evidenced after 6 weeks upon DIP supplementation at a high dose $(\mathrm{p}<0.0001)$ and 7 weeks of DIP administration at a low dose $(\mathrm{p}<0.01)(\mathrm{p}<0.001)$ (Figures 2A, B). Moreover, epididymal and subcutaneous fat accumulation was significantly reduced in DIP treated groups in a dose-dependent manner (Figures 2D, E). A significant increase in liver weight was witnessed in the HFD group $(\mathrm{p}<0.01)$ as compared to the control group. However, a substantial reduction in liver weight was observed at a high dose of DIP ( $\mathrm{p}<$ $0.01)$, and HFD + DIP(L) group didn't reach statistical significance (Figure 2F). These findings indicate that DIP decreases weight gain and fat accumulation in HFD-fed mice. Furthermore, food intake and energy intake were assessed in our study (Supplementary Figure S1). No significant difference was found among HFD alone and HFD followed by DIP treated groups, hence, suggesting the effect of DIP
A

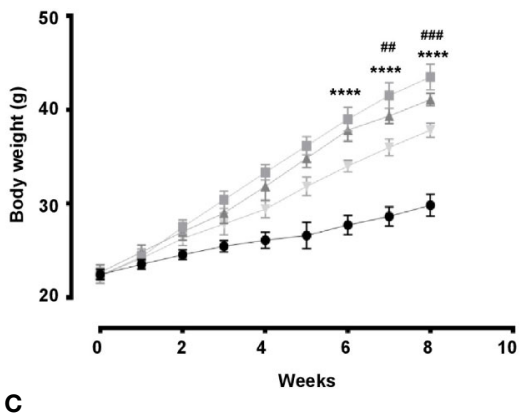

C

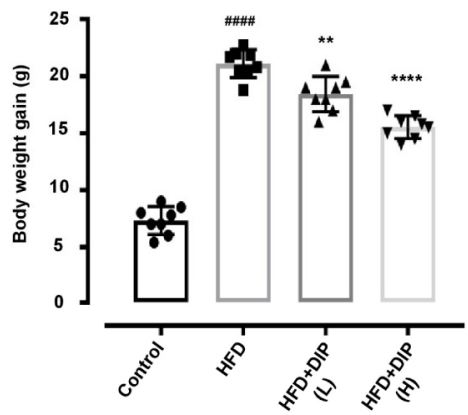

E

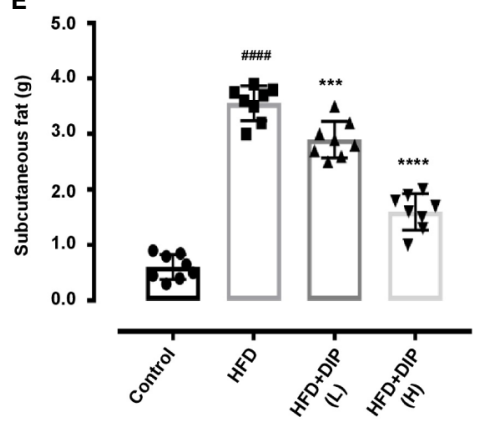

B
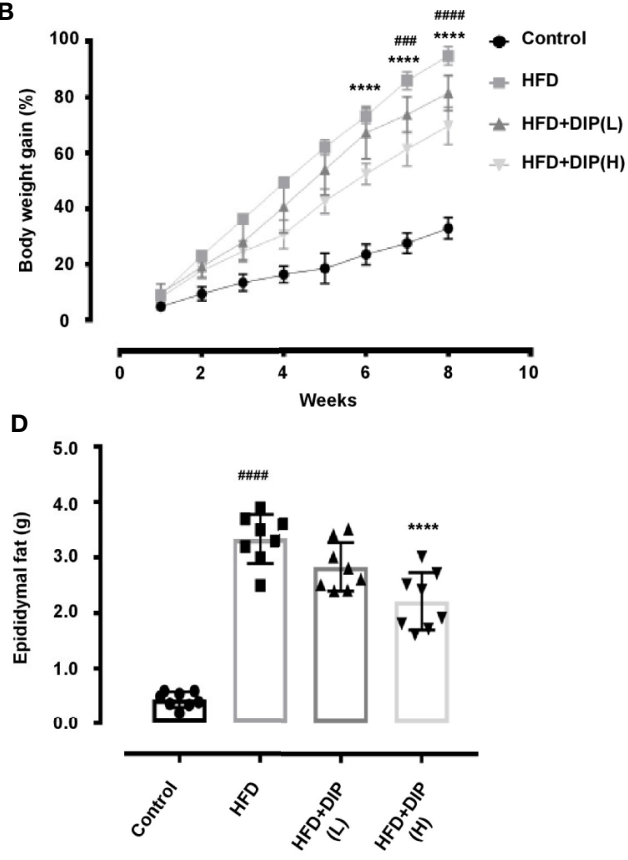

$\mathbf{F}$

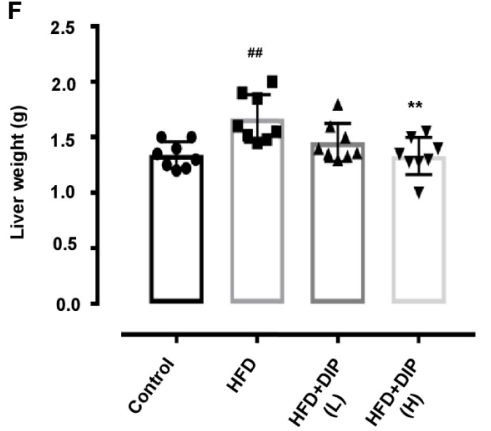

FIGURE 2 | Effect of DIP on body weight and fat accumulation in HFD fed mice. HFD-induced obese mice were treated with $200 \mu \mu$ of DIP at various doses for 4 weeks ( $\mathrm{n}=8$ each group). (A) Effects of DIP administration on body weight (g), (B) body weight gain (\%), (C) body weight gain (g), (D) epididymal fat (g), (E) subcutaneous fat (g), and (F) liver weight are presented in the above panels. Data are expressed as mean \pm SEM. Body weight differences in (A, B) were analyzed using two-way ANOVA

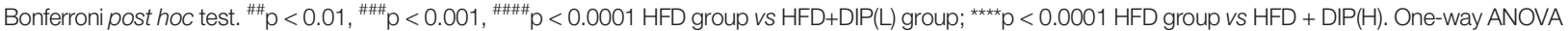

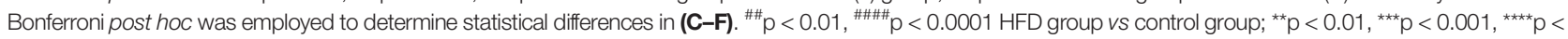
0.0001 HFD group vs HFD + DIP(L) and HFD + DIP(H) groups. 
on obesity-related parameters is not because of less intake of food or energy acquisition. Thus, these findings demonstrate that DIP has the potency to reverse HFD-induced obesity related parameters.

\section{Histological Examination of Epididymal Adipose Tissue, Determination of Adipogenic and Lipogenic Gene Expression Upon DIP Supplementation}

Since body weight gain was reduced after supplementation with DIP in HFD fed mice, we further determined if the bodyweight reduction was due to the decreased fat accumulation. Thus, the weight of epididymal and subcutaneous adipocytes was measured (Figures 2D, E), and hematoxylin and eosin (H\&E) staining was carried out to examine the histological alteration, i.e., adipocyte size in HFD fed versus HFD + DIP treated mice (Figure 3). Our finding revealed a significant increase of epididymal and subcutaneous fat accumulation, as presented in Figures 2D, E. Moreover, the histological analysis revealed the degree of lipid accumulation in the epididymal adipose tissue, which is proportional to the size of the tissue. The distribution of adipocytes in the HFD group depicted larger adipocyte size as compared to the control group. On the contrary, DIP treatment at a low dose and high dose $(\mathrm{p}<0.0001)$ depicted a significant adipocyte size reduction as compared to the HFD group suggesting the regulatory effect of DIP in fat deposition (Figure 3B). These findings were in agreement with previous studies that have reported a higher magnitude of lipid accumulation in HFD fed mice $(58,74)$.

Multiple research studies have shown that the key regulators of adipogenesis include peroxisome proliferator activated receptor $\gamma$ $(\mathrm{PPAR}-\gamma), \mathrm{CCAAT} / \mathrm{enh}$ ancer-binding protein alpha $(\mathrm{C} / \mathrm{EBP} \alpha)$, and sterol response element-binding protein-1c (SREBP-1c) genes (15, 75-78). The overexpression of these genes promotes adipogenesis and induces lipid accumulation. To elucidate the changes at transcriptional level, we determined the expression of these genes in HFD treated mice. In concordance with the earlier findings, our study has shown that expression level of PPAR- $\gamma, \mathrm{C} / \mathrm{EBP} \alpha$, and SREBP-1c is significantly enhanced in the HFD alone group compared to the control group (Figure 3C). However, DIP treatment at low dose $(\mathrm{p}<0.001)$ and at high dose $(\mathrm{p}<0.0001)$ regulated these transcription factors via regulation of the adipogenic genes. Furthermore, accumulated studies have suggested that the lipid biosynthesis associated genes including acetyl-CoA carboxylase1 (ACC-1) and fatty acid synthase (FAS) expression is higher in adipose and liver tissue in HFD fed obese mice $(15,79)$. Henceforth, we determined the relative expression of these lipogenic genes in the adipose tissue (Figure 3D). Our results revealed that the expression level of ACC-1 and FAS significantly increased in the HFD alone group ( $\mathrm{p}<0.001, \mathrm{p}<0.0001$ ) compared to the control group. Notwithstanding, DIP treatment at low dose $(\mathrm{p}<0.01)$ and at high dose $(\mathrm{p}<0.001, \mathrm{p}<0.0001)$ significantly decreased the expression of lipogenic genes.

\section{DIP Alleviated Liver Associated Markers and Lipid Profile of HFD-Induced Obesogenic Mice}

Previously, studies have shown that the HFD-induced obesity leads to higher serum alanine transaminase (ALT), aspartate transaminase (AST), triglycerides (TGs) and free fatty acid (FFA) levels $(15,80,81)$. Therefore, we investigated the effect of DIP intervention on ALT, AST, TG and FFA (Figures 4A-D). Our findings revealed that the HFD resulted in significant increase of serum ALT, AST, TG and FFA $(\mathrm{p}<0.0001)$ in the HFD alone group as compared to the control group (Figure 4). However, DIP treatment ameliorated liver health by reducing ALT $(p<0.01)$ DIP + HFD $(\mathrm{L}),(\mathrm{p}<0.0001)$ DIP $+\operatorname{HFD}(\mathrm{H})$, and AST levels $(\mathrm{p}<$ $0.001) \mathrm{DIP}+\operatorname{HFD}(\mathrm{L}),(\mathrm{p}<0.0001) \mathrm{DIP}+\operatorname{HFD}(\mathrm{H})$. Moreover, lipid markers such as TG $(\mathrm{p}<0.01)$ DIP $+\operatorname{HFD}(\mathrm{L}),(\mathrm{p}<0.0001)$ $\mathrm{DIP}+\operatorname{HFD}(\mathrm{H})$, and FFA $(\mathrm{p}<0.001) \mathrm{DIP}+\operatorname{HFD}(\mathrm{H})$ were also attenuated significantly. Altogether, our results infer that DIP attenuates liver and lipogenic markers in obesogenic mice.

\section{DIP Supplementation Ameliorated Serum LPS, Glucose, and Insulin Levels and Improves Intestinal Tight Junction Protein (TJP) Expression in HFD-Induced Obese Mice}

Previously, studies have reported that the HFD-induced obesity leads to higher serum lipopolysaccharide (LPS) levels $(13,15)$. Therefore, we investigated the effect of DIP intervention on serum endotoxin levels (Figure 5A). Our findings revealed that HFD resulted in elevated serum endotoxin levels $(\mathrm{p}<0.0001)$ in the HFD group as compared to the control group (Figure 5A). However, DIP treatment led to a significant reduction of LPS levels in DIP + HFD(L) ( $p<$ $0.01)$ and DIP $+\operatorname{HFD}(\mathrm{H})(\mathrm{p}<0.0001)$ groups. Moreover, glucose and insulin levels were also elevated in obesity $(58,74,82,83)$. To determine DIP outcomes on glucose homeostasis, we assessed fasting glucose and insulin levels. Our findings demonstrated a significant increase in fasting glucose and insulin levels in HFD alone group as compared to the control group (Supplementary Figure S2). Nonetheless, upon supplementation with DIP, glucose and insulin levels were reduced markedly.

The fundamental role of tight junction proteins in maintaining mucosal integrity is widely recognized, given that higher endotoxin levels increase the intestinal permeability and disrupt mucosal integrity (15). Hence, we comprehensively analyzed the tight junction protein (TJP) expression including claudin-1, occludin, and zonula occluden (ZO-1) of four experimental groups (Figures $\mathbf{5 B}, \mathbf{C})$. Immunoblot analysis was performed to demonstrate the robust alterations in TJP expression. The HFD alone group presented decreased TJP expression as compared to the control group (Figures 5B, C). However, after DIP intervention, the expression patterns of claudin-1, occludin, and zonula occluden (ZO-1) were upregulated significantly at high dose, and partial modulatory effect was observed at a low dose. Conclusively, our data suggest that HFD-induced obesity resulted in increased mucosal permeability and reduced TJP expression; nonetheless, DIP supplementation had a modulatory effect on intestinal integrity via decreasing endotoxin levels and increasing TJP expression.

\section{DIP Supplementation Reduced Inflammatory Cytokines in Obese Mice}

Previously, in-vivo studies have suggested that pro-inflammatory cytokine secretion is elevated in obese animals as compared to non-obese animals (58). Moreover, the upsurge of pro- 
A
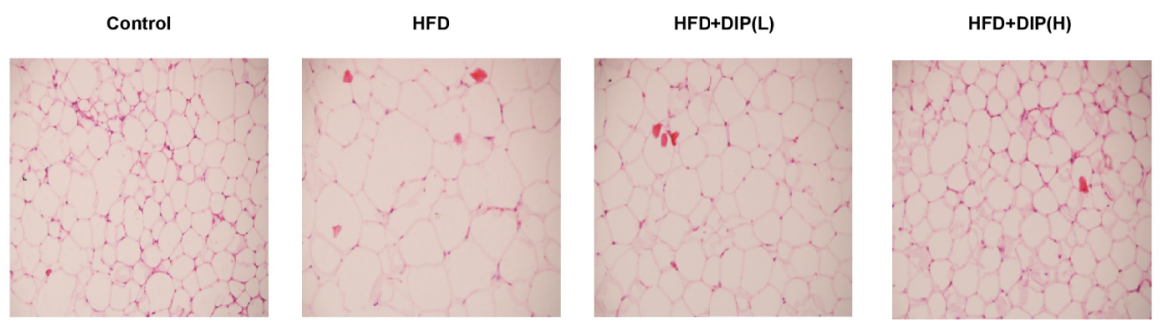

B

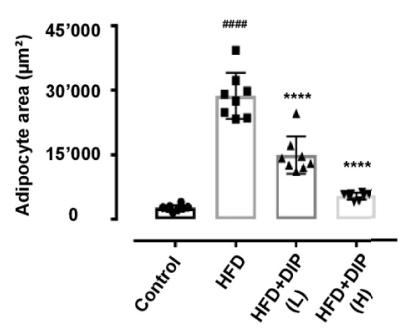

C
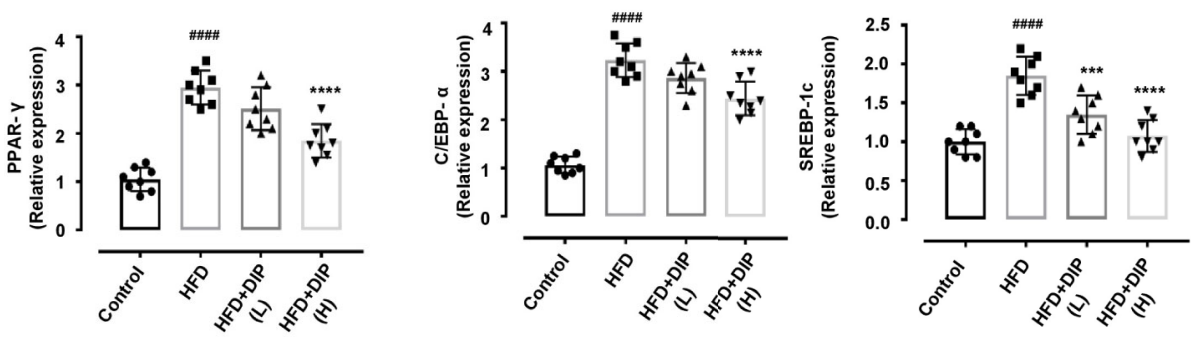

D
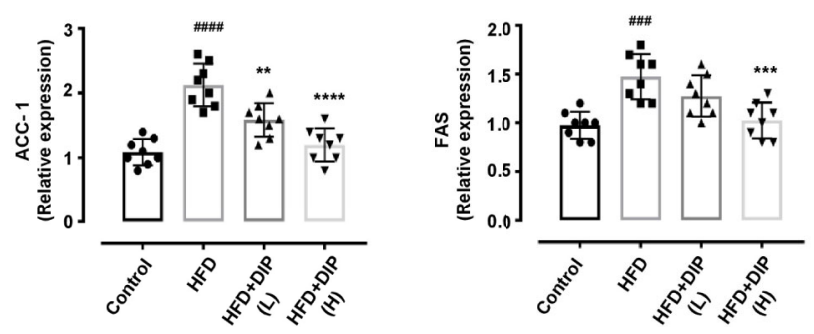

FIGURE 3 | Histologic analysis and lipogenic gene expression of epididymal adipose tissue in HFD-induced obese mice. Adipocyte structural analysis and size estimation through H\&E staining and ImageJ software. Scale bar, $50 \mu \mathrm{m}$. (A) The differences in the histology of adipocytes in various treatment groups are evident in representative H\&E stained images. (B) The distribution of adipocytes area indicates HFD has greater adipocytes area $\left(>20,000 \mu m^{2}\right)$. On the contrary, DIP treated groups revealed smaller adipocytes size $\left(<20,000 \mu \mathrm{m}^{2}\right)$. Data are expressed as the mean $\pm \mathrm{SEM},(\mathrm{n}=8)$ in each group based on one-way ANOVA Tukey post hoc

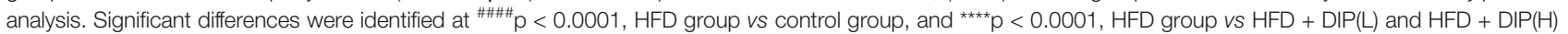
groups. (C) Effect of DIP supplementation treatment on the adipogenic genes expression PPAR- $\gamma, \mathrm{C} / \mathrm{EBP} \alpha$, and SREBP-1c in adipose tissues was determined with qRT-PCR. (D) Effect of DIP supplementation treatment on the lipogenic gene expression ACC-1, and FAS in adipose tissues was determined with qRT-PCR. The data is expressed as mean \pm SEM $(n=8)$ based on one-way ANOVA Tukey post hoc analysis. Significant differences were identified at ${ }^{\# \# \#} \mathrm{p}<0.001$, \#\#\#\# $\mathrm{p}<$ 0.0001 , HFD group vs control group; ${ }^{* \star} \mathrm{p}<0.01,{ }^{* \star *} \mathrm{p}<0.001$ and ${ }^{* \star * *} \mathrm{p}<0.0001, \mathrm{HFD}$ group $v s \mathrm{HFD}+\mathrm{DIP}(\mathrm{L})$ group or HFD group vs HFD + DIP(H) group.

inflammatory cytokines results in chronic inflammation and insulin resistance $(7,16,58,84)$. Hence we measured the level of pro-inflammatory including tumor necrosis factor- $\alpha$ (TNF$\alpha$ ), interleukin (IL-1 $\beta$ ), (IL-6) and anti-inflammatory cytokines (IL-4, IL-10) (Figure 6). In concordance with the previous findings, we also found that TNF- $\alpha$, IL- $1 \beta$, and IL-6 levels were significantly enhanced in the HFD alone group as compared to the control group. On the contrary, antiinflammatory cytokine secretion, such as IL-4 and IL-10 levels, was reduced in the HFD group. Nonetheless, DIP treatment significantly reduced the level of pro-inflammatory cytokines and enhanced anti-inflammatory cytokines in DIP supplemented 
A

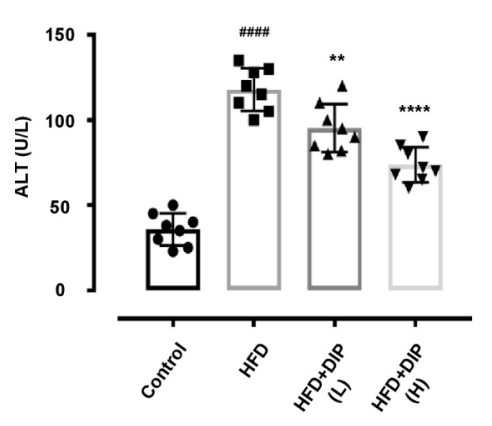

C

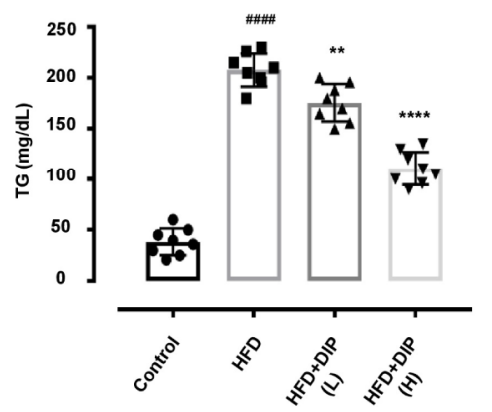

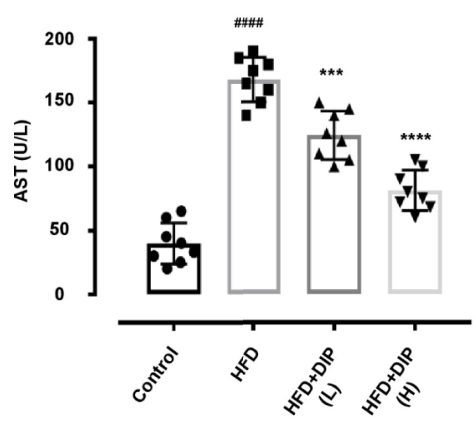

D

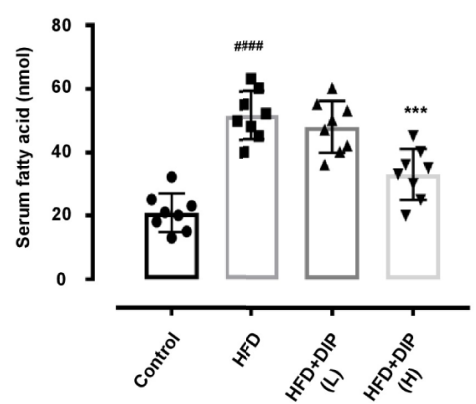

FIGURE 4 | DIP supplementation decreases serum ALT, AST, TG and FFA. Effect of DIP supplementation on serum (A) ALT, (B) AST, (C) TG and (D) FFA levels. The data is shown as mean \pm SEM $(n=8)$, based on one-way ANOVA Tukey post hoc analysis. Significant differences were identified using at \#\#\#\# $<$ <.0001 HFD group vs control group and ${ }^{* \star} p<0.01,{ }^{\star * *} p<0.001$ and ${ }^{\star \star \star *} p<0.0001$, HFD group vs HFD+DIP(L) and HFD+DIP(H) groups.

groups in a dose-dependent manner (Figure 6). The results revealed that DIP supplementation decreases the inflammatory markers in the obesogenic mice model.

\section{DIP Administration Reduced Inflammatory Markers in Epididymal Adipose Tissue and Liver of Obese Mice}

Multiple studies have demonstrated that adipose tissue of an obese animal has shown higher expression of pro-inflammatory cytokines such as TNF- $\alpha$, IL- $1 \beta$, and IL-6 levels $(16,58)$. We assessed the mRNA expression of these inflammatory cytokines in epididymal adipose tissue and liver. In harmony with the previous studies, we found a significantly higher expression level of TNF- $\alpha$, IL- $1 \beta$, and IL-6 in the HFD group in comparison with the control group (Figures 7A-C). The relative expression of these inflammatory cytokines was notably reduced by DIP treatment in a dosedependent manner ( $\mathrm{p}<0.05, \mathrm{p}<0.01, \mathrm{p}<0.0001)$ (Figures 7A-C).

Monocyte chemoattractant protein-1 (MCP-1) is a proinflammatory cytokine that has been linked with chronic inflammation through activation of M1 macrophages in obese mice models $(85,86)$. Henceforth, we determined the mRNA level of MCP-1in the epididymal adipose tissue and liver. In agreement with the earlier studies, we also observed a statistically significant higher mRNA expression of the MCP-1in the HFD group compared to the control group. However, DIP treatment significantly reduced MCP-1 expression levels at low and high doses $(\mathrm{p}<0.01, \mathrm{p}<0.001, \mathrm{p}<0.0001)$ (Figure 7D). Thus our findings revealed that DIP supplementation remarkably reduced inflammatory markers and macrophage infiltration in a dosedependent manner in HFD treated mice.

\section{DIP Supplementation Restored Gut Microbiota in Obese Mice}

Several reports have shown the intestinal microbiome perturbation (dysbiosis) in obesity, often characterized by reduced bacterial diversity and abundance, along with the alteration of the gut microbiota composition, i.e., increased Firmicutes to Bacteroidetes ratio $(15,42,87)$. In order to determine the effect of DIP intervention in HFD-induced obese mice, Illumina MiSeq sequencing of $16 \mathrm{~S} r \mathrm{RNA}$ (V3-V5) has been employed in this study. A total of 9,998 operational taxonomic units (OTUs) ranging from 520 to 756 were obtained. The total sequence read count was $1,644,813$, and after quality filtering, 1,339,583 clean sequencing reads were acquired. All the samples attained deep coverage (coverage $=1)$ (Supplementary Table S2). The alpha and beta diversity indices have been assessed to demonstrate the bacterial diversity, species richness, and similarities or dissimilarities between different treatment groups. The rank abundance curve depicting species diversity and richness in descending order were as follows: The HFD + DIP(H) group, control group, HFD group, and HFD + 


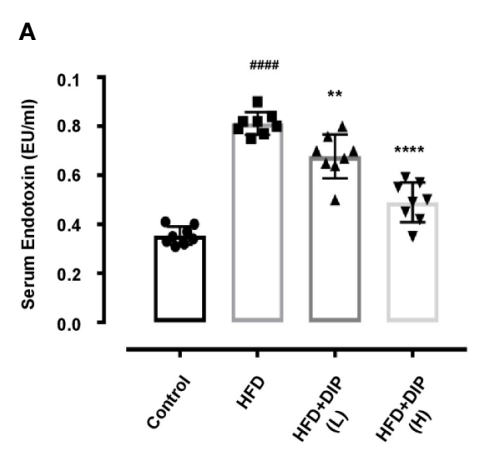

B

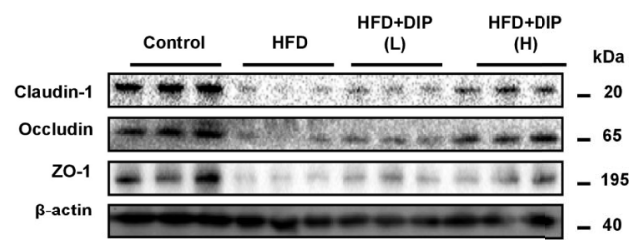

C
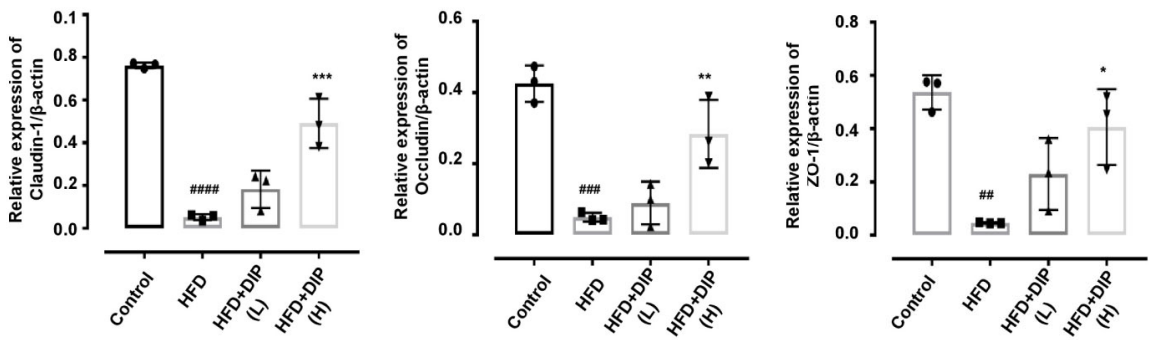

FIGURE 5 | DIP administration reduced serum endotoxin levels and ameliorated intestinal tight junction protein (TJP) expression in HFD fed mice. (A) Effect of DIP supplementation on serum LPS levels. Serum endotoxin (EU/ml) was determined using the limulus amebocyte lysate assay kit. The data is shown as mean \pm SEM $(\mathrm{n}=8)$, based on one-way ANOVA Tukey post hoc analysis. Significant differences were identified at ${ }^{\# \# \# p}<0.0001$ HFD group vs control group and ${ }^{* *} p<0.01$, ${ }_{* \star \star \star} \mathrm{p}<0.0001, \mathrm{HFD}$ group vs HFD + DIP(L) and HFD + DIP(H) groups. (B) Immunoblots presenting TJP expression in different treatment groups from the colon tissues. (C) Bar graph demonstrating relative band intensity of respective protein quantified via $\mathrm{NIH}$ image $\mathrm{J}$ software. The data was acquired from three independent

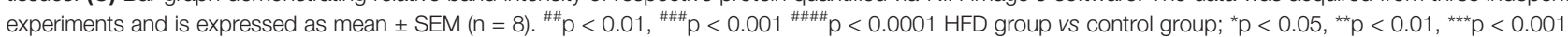
HFD group vs HFD + DIP(L) or HFD + DIP(H) groups.

DIP(L) group (Figure 8A). Meanwhile, rarefaction curves (Shannon index and observed species) show a saturation plateau with different depths for the respective group (Figures 8B-E). The higher species richness and abundance were witnessed for the control and HFD + DIP $(\mathrm{H})$ groups followed by the HFD + DIP (L) group. The Shannon index of the HFD group was significantly lower than the control and HFD $+\operatorname{DIP}(\mathrm{H})$ group $(\mathrm{p}<0.0001)$ (Figure 8C). The HFD group displayed the lowest observed species level as compared to the control and DIP treated groups (Figures 8D, E). However, no statistical difference was witnessed in observed species for all the experimental groups. Furthermore, $\beta$-diversity parameters were analyzed to measure the distance between each sample and the similarities between four experimental groups. The cluster dendrogram and principal coordinate analysis (PCoA) based on unweighted UniFrac distances depicted an evident clustering of bacterial taxa for each treatment group (Figures 8F, G).
The control and DIP treated groups showed apparent clustering depicting more close association and similarity in comparison with the HFD group. The analysis of similarity (ANOSIM) test using Bray-Curtis dissimilarity revealed that the observed cluster patterns were significant $(R=0.4722, P=0.002)$.

\section{HFD-Induced Bacterial Taxa Alteration Were Restored Upon DIP Administration}

At the phylum level, a total of ten bacterial phyla were detected, and among them, there were five dominated phyla: Firmicutes, Bacteroidetes, Proteobacteria, Actinobacteria, and TM7 (Figure 9A) (Supplementary Table S5). Previous studies have shown an increment of Firmicutes over Bacteroidetes in obese patients and HFD-induced obese mice (42, 87-89). In concordance with these studies, we also observed a relative increase abundance of Firmicutes to Bacteroidetes ratio in the HFD alone group as 

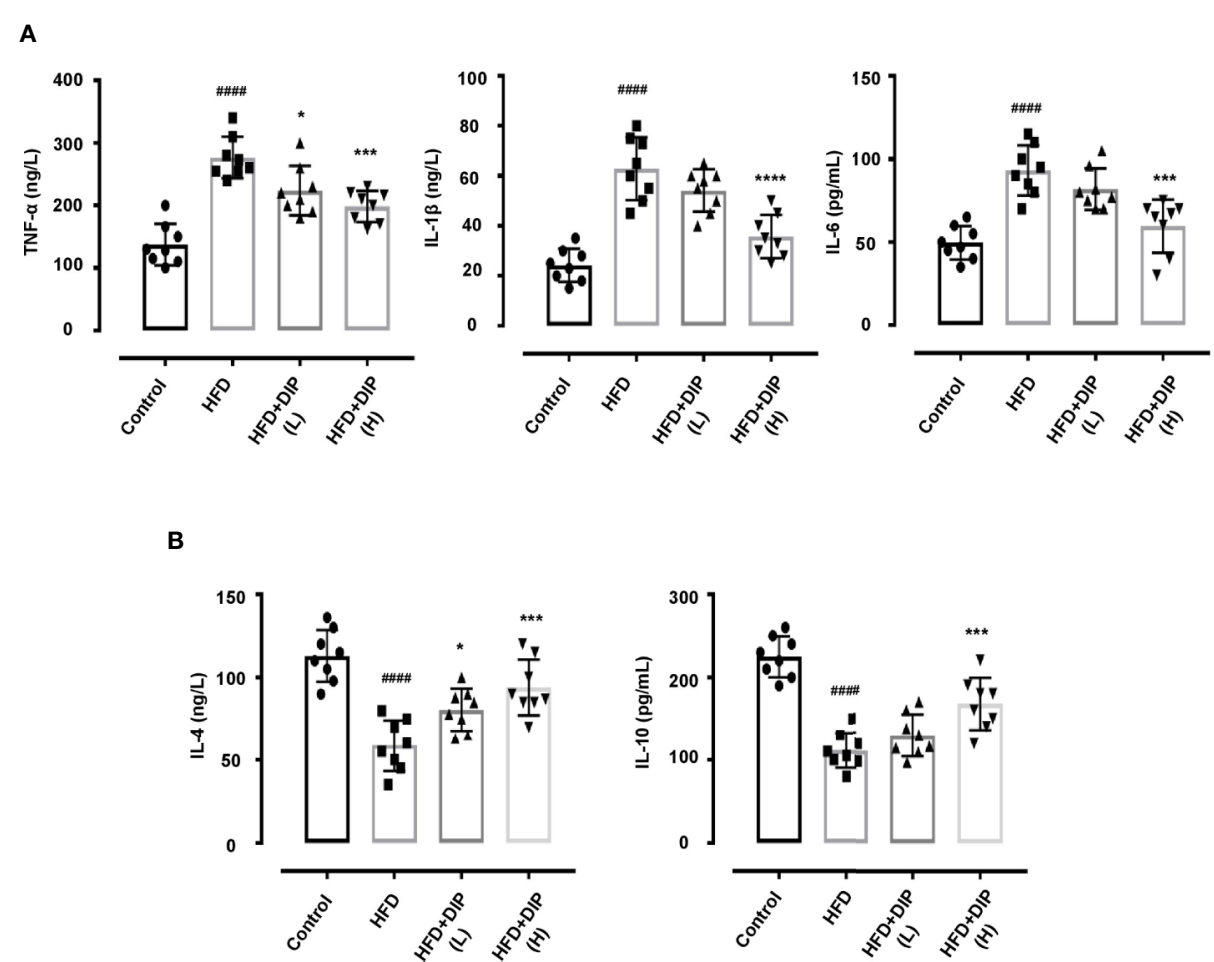

FIGURE 6 | Effect of DIP on pro-inflammatory and anti-inflammatory cytokines in the serum. (A) Pro-inflammatory cytokines TNF- $\alpha$, IL-6, IL-1 $\beta$. (B) Anti-inflammatory cytokines IL-4 and IL-10 levels in the serum were analyzed by ELISA kits. The data was acquired from three independent experiments and is presented as mean \pm SEM (n = 8). ${ }^{\# \# \# ~} \mathrm{p}<0.0001 \mathrm{HFD}$ group vs control group; ${ }^{\star} \mathrm{p}<0.05,{ }^{\star \star \star} \mathrm{p}<0.001$ and ${ }^{\star \star \star *} \mathrm{p}<0.0001 \mathrm{HFD}$ group vs HFD + DIP(L) or HFD + DIP(H) groups.

compared to the control group (Figure 9A). The higher abundance of Firmicutes and decreased abundance of Bacteroidetes were statistically significant in the HFD group in comparison with the control group $(\mathrm{p}<0.05)$ (Figure 9B). It is noteworthy that the bacterial taxonomy at the phylum level was reversed after DIP treatment, particularly at a high dose. The level of Firmicutes decreased, and Bacteroidetes increased after DIP treatment partially at a low dose and markedly at a high dose showing a similar trend like the control subjects. However, statistical significance was not achieved at the given doses of DIP.

Moreover, dynamic alterations were observed at the class level, where the major phyla were Bacteroidia, Clostridia, Bacilli, Gammaproteobacteria, and Actinobacteria (Figure 9C) (Supplementary Table S6). The HFD alone group presented a higher abundance of Bacilli (belongs to Firmicutes phylum) and Gammaproteobacteria (belongs to Proteobacteria phylum) and lower levels of Bacteroidia (belongs to Bacteroidetes phylum). The lower level of Bacteroidia $(\mathrm{p}<0.05)$ and increment of Bacilli ( $p<0.01$ ) were statistically significant in the HFD group as compared to the control group (Figure 9D). However, DIP treatment led to the restoration of altered microbiome composition, particularly at a high dose, which presented similar bacterial composition to that of the control group that were fed a standard chow diet. Overall, our findings revealed that HFD led to gut microbiota dysbiosis. Nonetheless, given DIP treatment, the dynamic bacterial perturbations were restored.

\section{DISCUSSION}

Obesity is characterized by systemic inflammation and is associated with the etiology of multiple chronic illnesses such as diabetes, cardiovascular diseases, liver inflammation, metabolic disorders, and various tumors (6, 7, 14, 90-92). The prevalence of obesity is increasing immensely around the globe. Henceforth, researchers are trying to develop novel anti-obesity arsenals to combat and halt this rate. Multiple studies have reported the therapeutic potential and health-promoting properties of polysaccharides from natural dietary sources such as mushrooms, seaweed, cereals, and herbs (93-99). The role of polysaccharide from edible and medicinal mushrooms in treating various illnesses is well documented $(15,58,96)$. Among them, D. indusiata is an edible, as well as a medicinal mushroom that possesses several biological properties $(64,67$, 100-102). To date, limited research has been done on the biological effect of $D$. indusiata in reducing obesity. Therefore we have used HFD-induced obesity mouse model to unravel the effect of $D$. indusiata polysaccharide (DIP) in reducing the dietary-induced obesity via modulating intestinal microbiota and inflammatory-related signaling pathways. In this study, our findings demonstrated that DIP polysaccharide reduced HFD-induced obesity parameters and inflammatory cascades via restoring intestinal microbial shift, regulating inflammatory reactions, and modulating intestinal integrity. 
A

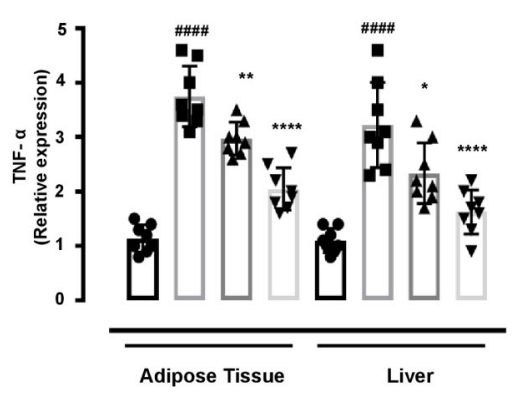

C

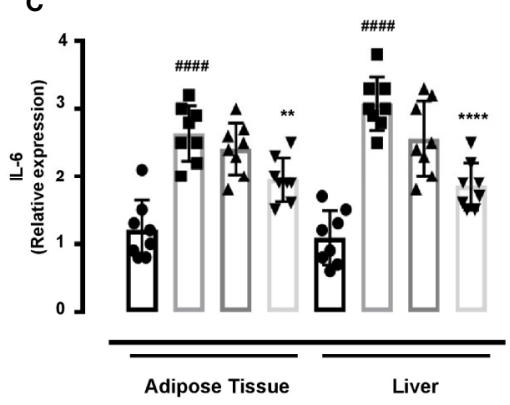

B

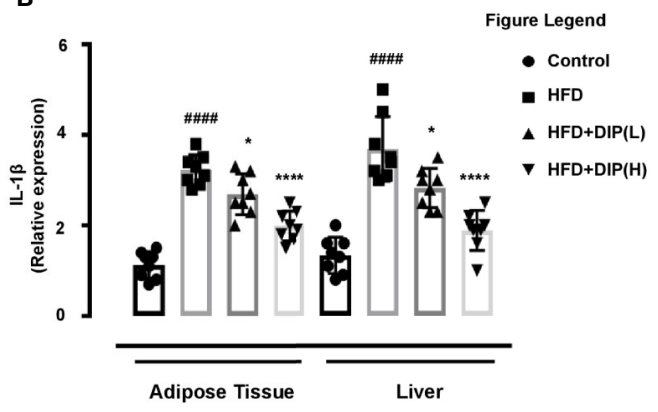

D

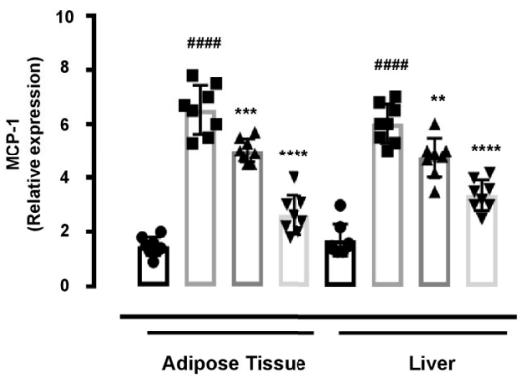

FIGURE 7 | Effect of DIP on adipokine expression in epididymal adipose tissue and liver. The relative mRNA expression of (A) TNF- $\alpha$ (B), IL-6, (C) IL-1 $\beta$, and (D) MCP-1 expression levels in the epididymal adipose tissue and hepatic tissue were analyzed with qPCR. The data is expressed as mean \pm SEM ( $n=8)$ based on

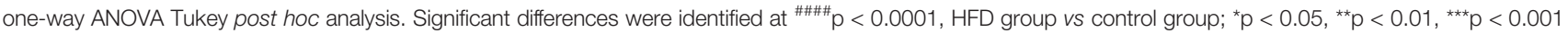
and ${ }^{\star \star \star *} \mathrm{p}<0.0001$, HFD group vs HFD + DIP(L) group or HFD group vs HFD + DIP(H) group.

Excessive lipid accumulation is one of the major factors in the development of obesity (103). In our study, HFD feeding led to a significant increment of body weight, epididymal and subcutaneous fat (Figure 2). Moreover, a substantial increase in liver weight was observed in the HFD alone group in comparison with the control group. However, DIP treatment reduced body weight gain significantly on the $7^{\text {th }}$ and $8^{\text {th }}$ weeks. Furthermore, fat deposition and liver weight were also reduced significantly at a high dose of DIP. These results indicate the protective effect of $D$. indusiata derived polysaccharide in reducing HFD-induced obesity parameters. Research literature shows that HFD-induced obesity often results in hyperinsulinemia and hyperglycemia $(15,104)$. As expected, our study showed elevated fasting glucose and insulin levels (Supplementary Figure S2). Interestingly, DIP supplementation at various doses attenuated the higher levels of glucose and insulin in HFD mice.

Several studies have suggested the enlarged epididymal adipose tissue size and lower number of adipocytes in obese individuals and in in-vivo studies $(15,105-107)$. Moreover, changes in the cascades of transcriptional factors, such as higher expression of adipogenic (PPAR- $\gamma, \mathrm{C} / \mathrm{EBP} \alpha$, SREBP-1c) and lipogenic genes (ACC-1, FAS) are documented in obesity associated studies $(15,75-79)$. In corroboration with these studies, we also found an increment of adipocytes size and increase expression of adipogenic and lipogenic genes in the HFD alone group in comparison with the control group. On the contrary, DIP treatment resulted in reducing adipocyte area and decreasing the expression of adipogenic genes in a dose-dependent manner (Figure 3). Additionally, hypertrophic adipocytes are attributed to secrete pro-inflammatory cytokines during the development of obesity $(108,109)$. The increased production of pro-inflammatory cytokines such as "TNF- $\alpha$, IL$1 \beta$, IL-6, and MCP- 1 " in turn, triggers chronic inflammatory reactions and enhance insulin resistance $(7,84)$. Thus, regulation of inflammatory cytokine production is one of the most critical factors to reduce inflammatory reactions and insulin resistance. In the present study, we observed a higher production of proinflammatory cytokines (TNF- $\alpha$, IL-1 $\beta$, IL- 6 , and MCP-1) (Figures 6 and 7) and lower levels of anti-inflammatory cytokines (IL-4, IL-10) (Figure 6) upon HFD feeding. Interestingly, DIP intervention for four weeks antagonized the inflammatory reactions by reducing pro-inflammatory cytokines and increasing antiinflammatory cytokines.

HFD feeding induces liver injury by elevating liver associated biomarkers such as (ALT and AST) and also enhances TG and FFA due to increased adiposity $(15,80,81,110)$. In agreement with the earlier findings, in our study, we also found higher levels of ALT, AST, TG, and FFA in HFD mice (Figure 4). Nonetheless, we observed that DIP reduced these markers in a dose-dependent manner suggesting the efficacy of DIP polysaccharide in ameliorating liver health and adiposity.

The higher level of pro-inflammatory cytokines as a result of high-fat diet intake are well known to trigger inflammatory reactions that tend to disrupt intestinal barrier, reduced TJP expression, and increased endotoxemia (LPS). The HFD induced 
A

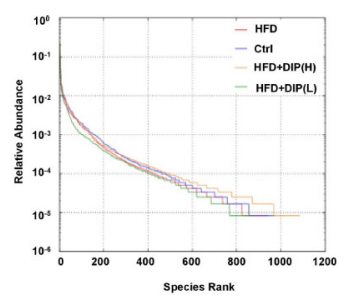

B

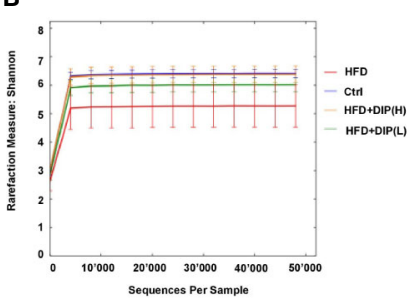

D

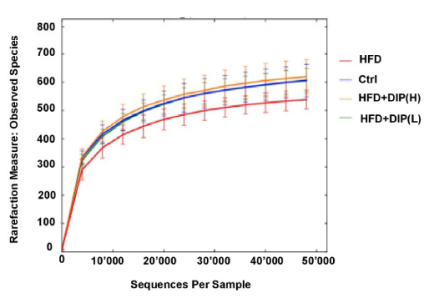

C

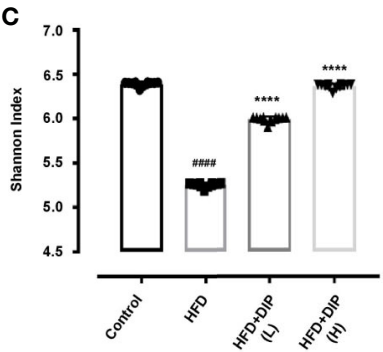

E

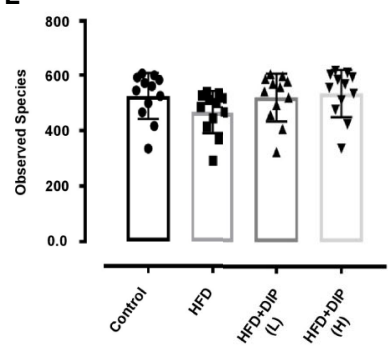

$\mathbf{F}$

Heigh

$\begin{array}{llllllll}0.06 & 0.08 & 0.10 & 0.12 & 0.14 & 0.16 & 0.18 & 0.20\end{array}$

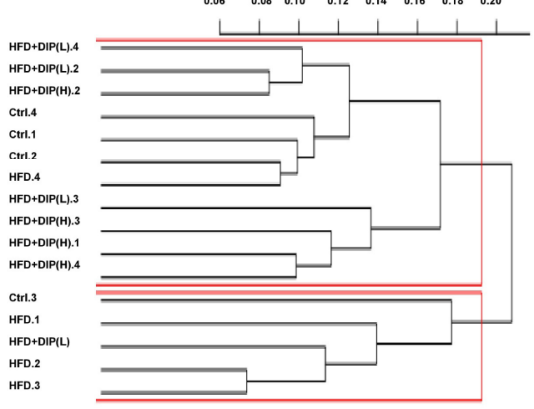

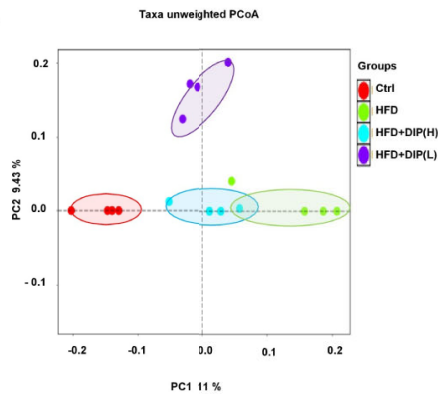

FIGURE 8 | Impact of DIP on gut microbiota diversity and abundance in HFD mice. (A) Rank abundance curve, (B) Shannon index, (C) bar graph for Shannon index, (D) observed species, (E) bar graph for observed species showing species diversity, richness, and abundance. (F) Cluster dendrogram and (G) principal coordinate analysis (PCOA) representing the level of similarities or dissimilarities among different treatment groups $(n=4)$. The data is presented as the mean \pm SEM, $(n=4)$ using one-way ANOVA

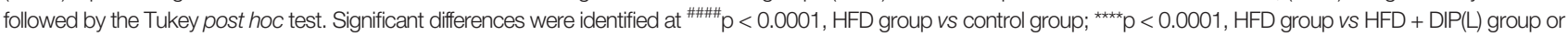
HFD group vs HFD + DIP(H) group. The clustering of PCoA analysis is statistically supported by the one-way ANOVA using ANOSIM test $(R=0.4722, p=0.002)$.

increased inflammatory cytokine production, and increased level of LPS levels in blood circulation leads to systemic and low grade inflammation via activation of the TLR4 signaling pathway (46). Our study demonstrated similar outcomes in HFD-induced obese mice. The endotoxin levels were quite enhanced in the HFD alone group comparatively to the control and DIP treated groups (Figure 5A). Moreover, TJP expression was downregulated in the HFD alone group (Figures $\mathbf{5 B}, \mathbf{C}$ ). On the 
A

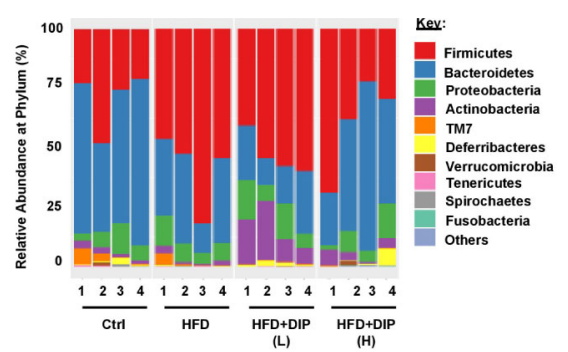

B
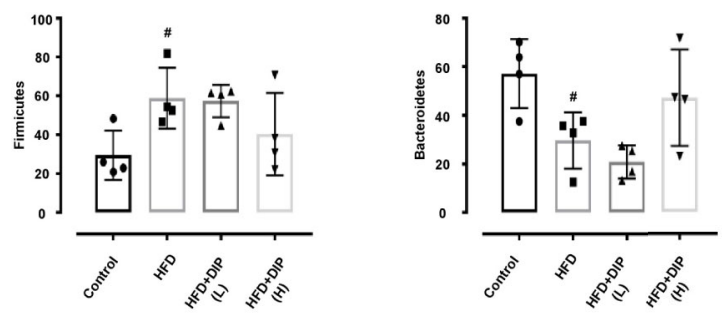

C

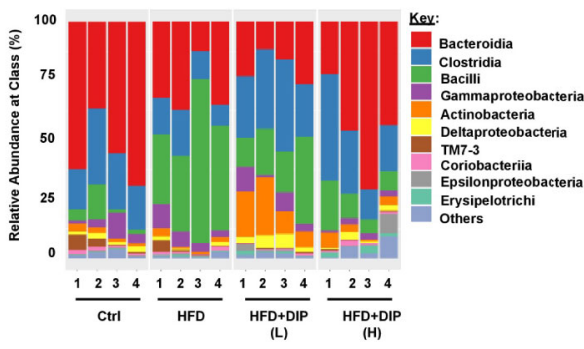

D
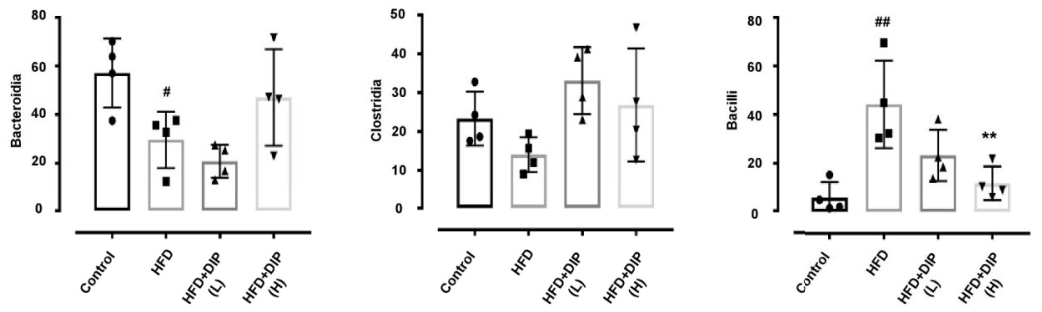

FIGURE 9 | The relative abundance of gut microbiota at phylum and class level in HFD mice. (A) The relative abundance of bacterial taxa at the phylum level. (B) Abundant flora at the phylum level (\%). (C) Relative abundance of bacterial taxa at the class level. (D) Abundant flora at the class level (\%). The data is shown as mean \pm SEM, $(n=4)$ using one-way ANOVA followed by the Tukey post hoc test. Significant differences were identified at ${ }^{\#} p<0.05,{ }^{\# \#} p<0.01$, HFD group vs control group, and ${ }^{* *} \mathrm{p}<0.01$, HFD group vs HFD + DIP(H) group.

contrary, DIP supplemented groups have shown decreased LPS levels and upregulated expression of TJPs compared to the HFD alone group. Altogether, these results suggested the modulatory effect of DIP supplementation in ameliorating intestinal integrity and inflammatory markers.

The intestinal microbiome is receiving much attention in recent years as studies have shown the association of gut microbiota in several inflammatory and obesity-related disorders (111-114). One of the most significant factors in obesity is the composition of the intestinal microbiome as gut microbes play an essential role in nutrient acquisition, vitamin production, energy, and fat storage regulation $(27,28,115)$. Therefore, to determine the link between gut microbiota and obesity may provide a much deeper insight into dealing with obesity-related health issues. Diet has a significant impact on the composition of the intestinal microbiome. HFD has been reported to cause an imbalance of intestinal microbiome composition in obese individuals, and the disruption of intestinal microbiome homeostasis has been linked to the progression of obesity and related metabolic disorders $(38,116)$. On the contrary, multiple studies have shown the modulatory effect offiber-rich diet 
such as prebiotics, polyphenols, oligosaccharides, and polysaccharides on gut microbiome community $(24,48,49,58$, 117). Manipulation of microbial flora through dietary fiber (prebiotics) and non-digestible oligosaccharides or polysaccharide can positively influence the microbial community by promoting the growth of beneficial flora $(118,119)$. To demonstrate the impact of polysaccharide treatment on gut microbiome community in HFD mice, we employed 16S rRNA Illumina miSeq sequencing. Our results showed that polysaccharide supplementation positively changed the signature of the intestinal microbiome in DIP intervention groups. On the contrary, we found reduced OTUs at all taxonomic levels in HFD alone group compared to the control and DIP treated groups (Supplementary Table S4). The alpha and beta diversities were assessed to determine the bacterial diversity, richness, abundance, and similarities among various treatment groups (Figure 8) (Supplementary Table S3). Previous studies have suggested lower bacterial diversity and richness in obese individuals in comparison with lean individuals $(41,120)$. The alpha diversity indices were measured with the rank abundance curve, Shannon index, and observed species. In agreement with the earlier findings, our study reflected similar outcomes, i.e., the HFD alone group presented the lowest bacterial diversity followed by HFD + DIP(L). The highest bacterial diversity was observed for the control and HFD + DIP( $\mathrm{H})$ group suggesting the beneficial effect of polysaccharide intervention. Moreover, the distribution pattern of microbial communities in various treatment groups presented as beta diversity indices (cluster dendrogram and PCoA) (Figures 8F, G). The cluster dendrogram and PCoA analysis revealed an obvious clustering of all the experimental groups, and the difference was statistically significant. The microbial composition in the control group and DIP intervention groups showed more closeness compared to the HFD alone group. HFD group clustered far from the control and DIP supplemented groups indicating the significance of polysaccharide ingestion in the diet as a modulatory compound for the bacterial community. Approximately $90 \%$ of the gut microbiota is composed of Firmicutes, Bacteroidetes, Proteobacteria, and Actinobacteria. Previous studies have shown alteration in the composition of these most abundant gut flora, i.e., higher Firmicutes to Bacteroidetes ratio in obese mice models (42, 87). In our study, Firmicutes, Bacteroidetes, Proteobacteria, Actinobacteria, TM7, and Deferribacteres were evidenced where Firmicutes and Bacteroidetes were the most abundant flora at Phylum level. At the class level, the major phyla were Bacteroidia, Clostridia, Bacilli, and Gammaproteobacteria. Overall, perturbation of bacterial flora was observed at Phylum and Class levels in the HFD group. In agreement with previous studies, we also found an increased abundance of Firmicutes and reduced Bacteroidetes levels in the HFD alone group (Figures 9A, B). Moreover, at the class level, the HFD group depicted an increased abundance of Bacilli, Gammaproteobacteria (belongs to Firmicutes and Proteobacteria) and decrease abundance of Bacteroidia (belongs to Bacteroidetes) compared to the control and DIP treated groups (Figures 9C, D). Interestingly HFD-induced bacterial alterations were reversed by DIP supplementation partially at low dose and greatly at a high dose, suggesting the modulatory impact of polysaccharide supplementation on gut microbiome composition.
In our research, we adopted the approach used by Liang $\mathrm{Z}$ et al., Lee H-G et al., and An Y et al. $(71,121,122)$ and therefore, we haven't randomized the treatment within each cage and kept each individual cage for one specific treatment i.e., one cage for the high dose treatment and another for the low dose treatment. However, randomizing the treatment within the cages could be another efficient approach, which can help to eliminate the cage/litter effect as well. Hence, we will adopt this methodology in future studies and will compare the outcomes with the reported results.

\section{CONCLUSIONS}

In conclusion, our study demonstrated that HFD supplementation for eight weeks led to obesity in mice that is associated with increased body weight, epididymal and subcutaneous fat accumulation, increase fasting glucose and insulin levels, elevated lipogenic gene expression, increase inflammatory reactions, such as high endotoxin (LPS) and pro-inflammatory cytokines secretion, reduced TJP expression and gut dysbiosis. D. indusiata derived crude polysaccharide (DIP) supplementation resulted in alleviating HFD-induced obesity by mitigating obesity related parameters. The inflammatory cascades, such as the increment of pro-inflammatory cytokines, elevated lipogenic genes expression and higher endotoxin levels, were regulated. The expression levels of TJPs were enhanced, and the inflammatory reactions were modulated. The bacterial taxa shift is corrected by reducing the abundance of bacterial taxa involved in obesity, such as Firmicutes, Clostridia, and Bacilli. Comprehensively, our findings suggested that D. indusiata possesses anti-obesogenic and anti-inflammatory properties. However, further research is needed on the purification and identification of the bioactive compounds of crude polysaccharide from $D$. indusiata that are responsible for mitigating obesity and associated parameters in a mice model as well as to test the effect of DIP in clinical trials.

\section{DATA AVAILABILITY STATEMENT}

The datasets used and analyzed during the current study are available from the corresponding author on reasonable request.

\section{ETHICS STATEMENT}

The animal study was reviewed and approved by Dalian Medical University Committee for animal experiments.

\section{AUTHOR CONTRIBUTIONS}

SK and YX conceived the study. YX attained funds and supervised the project. SK and SA designed the experiments. SK performed the experiments. SK acquired and analyzed the 
data. SK wrote the original article, which was revised critically by SA and YX. All authors contributed to the article and approved the submitted version.

\section{FUNDING}

This research was funded by the Chinese Scholarship Council grant number CSC no: 2016DFH376, and "The APC was supported by Liaoning Provincial Program for Top Discipline of Basic Medical Sciences".

\section{REFERENCES}

1. Hoyt CL, Burnette JL, Auster-Gussman L. "Obesity is a disease" examining the self-regulatory impact of this public-health message. Psychol Sci (2014) 25(4):997-1002. doi: 10.1177/0956797613516981

2. Bray GA, Frühbeck G, Ryan DH, Wilding JP. Management of obesity. Lancet (2016) 387(10031):1947-56. doi: 10.1016/S0140-6736(16)00271-3

3. Kelly T, Yang W, Chen C-S, Reynolds K, He J. Global burden of obesity in 2005 and projections to 2030. Int J Obes (2008) 32(9):1431-7. doi: 10.1038/ ijo.2008.102

4. Moustafa JSE-S, Froguel P. From obesity genetics to the future of personalized obesity therapy. Nat Rev Endocrinol (2013) 9(7):402. doi: 10.1038/nrendo. 2013.57

5. Barazzoni R, Bischoff S, Boirie Y, Busetto L, Cederholm T, Dicker D, et al. Sarcopenic obesity: Time to meet the challenge. Obes Facts (2018) 11 (4):294-305. doi: 10.1159/000490361

6. Yoshimoto S, Loo TM, Atarashi K, Kanda H, Sato S, Oyadomari S, et al. Obesityinduced gut microbial metabolite promotes liver cancer through senescence secretome. Nature (2013) 499(7456):97-101. doi: 10.1038/nature12347

7. Osborn O, Olefsky JM. The cellular and signaling networks linking the immune system and metabolism in disease. Nat Med (2012) 18(3):363. doi: $10.1038 / \mathrm{nm} .2627$

8. Wang Y, Zhu Y, Ruan K, Wei H, Feng Y. MDG-1, a polysaccharide from Ophiopogon japonicus, prevents high fat diet-induced obesity and increases energy expenditure in mice. Carbohydr Polym (2014) 114:183-9. doi: 10.1016/j.carbpol.2014.08.013

9. Segula D. Complications of obesity in adults: a short review of the literature. Malawi Med J (2014) 26(1):20-4.

10. Cox AJ, West NP, Cripps AW. Obesity, inflammation, and the gut microbiota. Lancet Diabetes Endocrinol (2015) 3(3):207-15. doi: 10.1016/ S2213-8587(14)70134-2

11. Ahmad R, Rah B, Bastola D, Dhawan P, Singh AB. Obesity-induces organ and tissue specific tight junction restructuring and barrier deregulation by claudin switching. Sci Rep (2017) 7(1):1-16. doi: 10.1038/s41598-017-04989-8

12. Brun $P$, Castagliuolo I, Leo VD, Buda A, Pinzani M, Palù G, et al. Increased intestinal permeability in obese mice: new evidence in the pathogenesis of nonalcoholic steatohepatitis. Am J Physiol Gastrointestinal Liver Physiol (2007) 292(2):G518-G25. doi: 10.1152/ajpgi.00024.2006

13. Cani PD, Bibiloni R, Knauf C, Waget A, Neyrinck AM, Delzenne NM, et al. Changes in gut microbiota control metabolic endotoxemia-induced inflammation in high-fat diet-induced obesity and diabetes in mice. Diabetes (2008) 57(6):1470-81. doi: 10.2337/db07-1403

14. Li H, Lelliott C, Håkansson P, Ploj K, Tuneld A, Verolin-Johansson M, et al. Intestinal, adipose, and liver inflammation in diet-induced obese mice. Metabolism (2008) 57(12):1704-10. doi: 10.1016/j.metabol.2008.07.029

15. Chang C-J, Lin C-S, Lu C-C, Martel J, Ko Y-F, Ojcius DM, et al. Ganoderma lucidum reduces obesity in mice by modulating the composition of the gut microbiota. Nat Commun (2015) 6(1):1-19. doi: 10.1038/ncomms8489

16. Cani PD, Amar J, Iglesias MA, Poggi M, Knauf C, Bastelica D, et al. Metabolic endotoxemia initiates obesity and insulin resistance. Diabetes (2007) 56(7):1761-72. doi: 10.2337/db06-1491

\section{ACKNOWLEDGMENTS}

We would like to thank Dr. Muhammad Ahmed Khan for editorial and technical assistance.

\section{SUPPLEMENTARY MATERIAL}

The Supplementary Material for this article can be found online at: https:/www.frontiersin.org/articles/10.3389/fendo.2020.558874/ full\#supplementary-material

17. Wellen KE, Hotamisligil GS. Inflammation, stress, and diabetes. J Clin Invest (2005) 115(5):1111-9. doi: 10.1172/JCI25102

18. Baranova IN, Souza AC, Bocharov AV, Vishnyakova TG, Hu X, Vaisman BL, et al. Human SR-BI and SR-BII potentiate lipopolysaccharide-induced inflammation and acute liver and kidney injury in mice. J Immunol (2016) 196(7):3135-47. doi: 10.4049/jimmunol.1501709

19. Odegaard JI, Chawla A. Pleiotropic actions of insulin resistance and inflammation in metabolic homeostasis. Science (2013) 339(6116):172-7. doi: $10.1126 /$ science. 1230721

20. Weisberg SP, McCann D, Desai M, Rosenbaum M, Leibel RL, Ferrante AW. Obesity is associated with macrophage accumulation in adipose tissue. J Clin Invest (2003) 112(12):1796-808. doi: 10.1172/JCI200319246

21. Fei N, Zhao L. An opportunistic pathogen isolated from the gut of an obese human causes obesity in germfree mice. ISME J (2013) 7(4):880-4. doi: 10.1038/ismej.2012.153

22. Liou A, Paziuk M, Luevano J. Conserved shifts in the gut microbiota due to gastric bypass reduce host weight and adiposity. Sci Transl Med (2013) 5 (178):178141. doi: 10.1126/scitranslmed.3005687

23. Bajzer M, Seeley RJ. Obesity and gut flora. Nature (2006) 444(7122):100910. doi: $10.1038 / 4441009$ a

24. Etxeberria U, Fernández-Quintela A, Milagro FI, Aguirre L, Martínez JA, Portillo MP. Impact of polyphenols and polyphenol-rich dietary sources on gut microbiota composition. J Agric Food Chem (2013) 61(40):9517-33. doi: $10.1021 / \mathrm{jf} 402506 \mathrm{c}$

25. Marchesi JR, Adams DH, Fava F, Hermes GD, Hirschfield GM, Hold G, et al The gut microbiota and host health: a new clinical frontier. Gut (2016) 65 (2):330-9. doi: 10.1136/gutjnl-2015-309990

26. Gibson GR, Scott KP, Rastall RA, Tuohy KM, Hotchkiss A, Dubert-Ferrandon A, et al. Dietary prebiotics: current status and new definition. Food Sci Technol Bull Funct Foods (2010) 7(1):1-19. doi: 10.1616/1476-2137.15880

27. Dethlefsen L, McFall-Ngai M, Relman DA. An ecological and evolutionary perspective on human-microbe mutualism and disease. Nature (2007) 449 (7164):811-8. doi: 10.1038/nature06245

28. Bäckhed F, Ding H, Wang T, Hooper LV, Koh GY, Nagy A, et al. The gut microbiota as an environmental factor that regulates fat storage. Proc Natl Acad Sci (2004) 101(44):15718-23. doi: 10.1073/pnas.0407076101

29. Belizário JE, Faintuch J. Microbiome and gut dysbiosis. In: Metabolic Interaction in Infection. Switzerland AG: Springer Nature (2018). p. 45976. doi: 10.1007/978-3-319-74932-7_13

30. Peterson CT, Sharma V, Elmén L, Peterson SN. Immune homeostasis, dysbiosis and therapeutic modulation of the gut microbiota. Clin Exp Immunol (2015) 179(3):363-77. doi: 10.1111/cei.12474

31. Rogers G, Keating D, Young R, Wong M, Licinio J, Wesselingh S. From gut dysbiosis to altered brain function and mental illness: mechanisms and pathways. Mol Psychiatry (2016) 21(6):738-48. doi: 10.1038/mp.2016.50

32. Tilg H, Moschen AR. Microbiota and diabetes: an evolving relationship. Gut (2014) 63(9):1513-21. doi: 10.1136/gutjnl-2014-306928

33. Rigottier-Gois L. Dysbiosis in inflammatory bowel diseases: the oxygen hypothesis. ISME J (2013) 7(7):1256-61. doi: 10.1038/ismej.2013.80

34. Kaur N, Chen C-C, Luther J, Kao JY. Intestinal dysbiosis in inflammatory bowel disease. Gut Microbes (2011) 2(4):211-6. doi: 10.4161/gmic.2.4.17863 
35. Kim D, Zeng MY, Núñez G. The interplay between host immune cells and gut microbiota in chronic inflammatory diseases. Exp Mol Med (2017) 49(5): e339. doi: 10.1038/emm.2017.24

36. de Sant'Ana LP, Ribeiro DJS, Martins AMA, dos Santos FN, Corrêa R, Almeida R, et al. Absence of the Caspases 1/11 Modulates Liver Global Lipid Profile and Gut Microbiota in High-Fat-Diet-Induced Obese Mice. Front Immunol (2020) 10:2926. doi: 10.3389/fimmu.2019.02926

37. Machado MV, Cortez-Pinto H. Diet, microbiota, obesity, and NAFLD: a dangerous quartet. Int J Mol Sci (2016) 17(4):481. doi: 10.3390/ijms17040481

38. Ridaura VK, Faith JJ, Rey FE, Cheng J, Duncan AE, Kau AL, et al. Gut microbiota from twins discordant for obesity modulate metabolism in mice. Science (2013) 341(6150):1241214. doi: 10.1126/science.1241214

39. Turnbaugh PJ, Ley RE, Mahowald MA, Magrini V, Mardis ER, Gordon JI. An obesity-associated gut microbiome with increased capacity for energy harvest. nature (2006) 444(7122):1027. doi: 10.1038/nature05414

40. Goodman AL, Kallstrom G, Faith JJ, Reyes A, Moore A, Dantas G, et al. Extensive personal human gut microbiota culture collections characterized and manipulated in gnotobiotic mice. Proc Natl Acad Sci (2011) 108 (15):6252-7. doi: 10.1073/pnas.1102938108

41. Ley RE, Bäckhed F, Turnbaugh P, Lozupone CA, Knight RD, Gordon JI. Obesity alters gut microbial ecology. Proc Natl Acad Sci (2005) 102 (31):11070-5. doi: 10.1073/pnas.0504978102

42. Daniel H, Gholami AM, Berry D, Desmarchelier C, Hahne H, Loh G, et al. High-fat diet alters gut microbiota physiology in mice. ISME J (2014) 8 (2):295-308. doi: 10.1038/ismej.2013.155

43. Turnbaugh PJ, Bäckhed F, Fulton L, Gordon JI. Diet-induced obesity is linked to marked but reversible alterations in the mouse distal gut microbiome. Cell Host Microbe (2008) 3(4):213-23. doi: 10.1016/ j.chom.2008.02.015

44. Burcelin R, Crivelli V, Dacosta A, Roy-Tirelli A, Thorens B. Heterogeneous metabolic adaptation of C57BL/6J mice to high-fat diet. Am J Physiol Endocrinol Metab (2002) 282(4):E834-42. doi: 10.1152/ajpendo.00332.2001

45. Kong LC, Holmes BA, Cotillard A, Habi-Rachedi F, Brazeilles R, Gougis S, et al. Dietary patterns differently associate with inflammation and gut microbiota in overweight and obese subjects. PloS One (2014) 9(10): e109434. doi: 10.1371/journal.pone.0109434

46. Shi H, Kokoeva MV, Inouye K, Tzameli I, Yin H, Flier JS. TLR4 links innate immunity and fatty acid-induced insulin resistance. J Clin Invest (2006) 116 (11):3015-25. doi: 10.1172/JCI28898

47. Ghanim H, Abuaysheh S, Sia CL, Korzeniewski K, Chaudhuri A, FernandezReal JM, et al. Increase in plasma endotoxin concentrations and the expression of Toll-like receptors and suppressor of cytokine signaling-3 in mononuclear cells after a high-fat, high-carbohydrate meal: implications for insulin resistance. Diabetes Care (2009) 32(12):2281-7. doi: 10.2337/dc09-0979

48. Delzenne NM, Bindels LB. Ganoderma lucidum, a new prebiotic agent to treat obesity? Nat Rev Gastroenterol Hepatol (2015) 12(10):553-4. doi: 10.1038/nrgastro.2015.137

49. Neyrinck AM, Possemiers S, Druart C, Van de Wiele T, De Backer F, Cani $\mathrm{PD}$, et al. Prebiotic effects of wheat arabinoxylan related to the increase in bifidobacteria, Roseburia and Bacteroides/Prevotella in diet-induced obese mice. PloS One (2011) 6(6):e20944. doi: 10.1371/journal.pone.0020944

50. Molinaro F, Paschetta E, Cassader M, Gambino R, Musso G. Probiotics, prebiotics, energy balance, and obesity: mechanistic insights and therapeutic implications. Gastroenterol Clinics (2012) 41(4):843-54. doi: 10.1016/ j.gtc.2012.08.009

51. Arora T, Singh S, Sharma RK. Probiotics: interaction with gut microbiome and antiobesity potential. Nutrition (2013) 29(4):591-6. doi: 10.1016/ j.nut.2012.07.017

52. Di Cagno R, Filannino P, Vincentini O, Cantatore V, Cavoski I, Gobbetti M. Fermented Portulaca oleracea L. Juice: A Novel Functional Beverage with Potential Ameliorating Effects on the Intestinal Inflammation and Epithelial Injury. Nutrients (2019) 11(2):248. doi: 10.3390/nu11020248

53. Filannino P, Tlais AZ, Morozova K, Cavoski I, Scampicchio M, Gobbetti M, et al. Lactic acid fermentation enriches the profile of biogenic fatty acid derivatives of Avocado fruit (Persea americana Mill.). Food Chem (2020) 317:126384. doi: 10.1016/j.foodchem.2020.126384

54. Kaukua J, Pekkarinen T, Sane T, Mustajoki P. Health-related quality of life in obese outpatients losing weight with very-low-energy diet and behaviour modification-a 2-y follow-up study. Int J Obes (2003) 27(10):1233-41. doi: 10.1038/sj.ijo.0802379

55. Kang JG, Park C-Y. Anti-obesity drugs: a review about their effects and safety. Diabetes Metab J (2012) 36(1):13-25. doi: 10.4093/dmj.2012.36.1.13

56. Christensen R, Kristensen PK, Bartels EM, Bliddal H, Astrup A. Efficacy and safety of the weight-loss drug rimonabant: a meta-analysis of randomised trials. Lancet (2007) 370(9600):1706-13. doi: 10.1016/S0140-6736(07)61721-8

57. Bindels LB, Delzenne NM, Cani PD, Walter J. Towards a more comprehensive concept for prebiotics. Nat Rev Gastroenterol Hepatol (2015) 12(5):303. doi: 10.1038/nrgastro.2015.47

58. Chang C, Lu C, Lin C, Martel J, Ko Y, Ojcius D, et al. Antrodia cinnamomea reduces obesity and modulates the gut microbiota in high-fat diet-fed mice. Int J Obes (2018) 42(2):231-43. doi: 10.1038/ijo.2017.149

59. Gibson GR, Probert HM, Van Loo J, Rastall RA, Roberfroid MB. Dietary modulation of the human colonic microbiota: updating the concept of prebiotics. Nutr Res Rev (2004) 17(2):259-75. doi: 10.1079/NRR200479

60. Rathore H, Prasad S, Sharma S. Mushroom nutraceuticals for improved nutrition and better human health: A review. PharmaNutrition (2017) 5 (2):35-46. doi: 10.1016/j.phanu.2017.02.001

61. Cani PD, Neyrinck AM, Fava F, Knauf C, Burcelin RG, Tuohy KM, et al. Selective increases of bifidobacteria in gut microflora improve high-fat-dietinduced diabetes in mice through a mechanism associated with endotoxaemia. Diabetologia (2007) 50(11):2374-83. doi: 10.1007/s00125-007-0791-0

62. Belkaid Y, Hand TW. Role of the microbiota in immunity and inflammation. Cell (2014) 157(1):121-41. doi: 10.1016/j.cell.2014.03.011

63. Cani PD, Possemiers S, Van de Wiele T, Guiot Y, Everard A, Rottier O, et al. Changes in gut microbiota control inflammation in obese mice through a mechanism involving GLP-2-driven improvement of gut permeability. Gut (2009) 58(8):1091-103. doi: 10.1136/gut.2008.165886

64. Wang Y, Shi X, Yin J, Nie S. Bioactive polysaccharide from edible Dictyophora spp.: Extraction, purification, structural features and bioactivities. Bioactive Carbohydrates Dietary Fibre (2018) 14:25-32. doi: 10.1016/j.bcdf.2017.07.008

65. Hua Y, Gao Q, Wen L, Yang B, Tang J, You L, et al. Structural characterisation of acid-and alkali-soluble polysaccharides in the fruiting body of Dictyophora indusiata and their immunomodulatory activities. Food Chem (2012) 132(2):739-43. doi: 10.1016/j.foodchem.2011.11.010

66. Liu X, Chen Y, Wu L, Wu X, Huang Y, Liu B. Optimization of polysaccharides extraction from Dictyophora indusiata and determination of its antioxidant activity. Int J Biol Macromolecules (2017) 103:175-81. doi: 10.1016/j.ijbiomac.2017.04.125

67. Han S, Ma C, Hu M, Wang Y, Ma F, Tao N, et al. A polysaccharide from Dictyophora indusiata inhibits the immunosuppressive function of cancerassociated fibroblasts. Cell Biochem Funct (2017) 35(7):414-9. doi: 10.1002/ cbf. 3290

68. Wang Y, Lai L, Teng L, Li Y, Cheng J, Chen J, et al. Mechanism of the antiinflammatory activity by a polysaccharide from Dictyophora indusiata in lipopolysaccharide-stimulated macrophages. Int J Biol Macromolecules (2019) 126:1158-66. doi: 10.1016/j.ijbiomac.2019.01.022

69. Kanwal S, Joseph TP, Owusu L, Xiaomeng R, Meiqi L, Yi X. A polysaccharide isolated from Dictyophora indusiata promotes recovery from antibiotic-driven intestinal dysbiosis and improves gut epithelial barrier function in a mouse model. Nutrients (2018) 10(8):1003. doi: $10.3390 /$ nu10081003

70. Kanwal S, Joseph TP, Aliya S, Song S, Saleem MZ, Nisar MA, et al. Attenuation of DSS induced colitis by Dictyophora indusiata polysaccharide (DIP) via modulation of gut microbiota and inflammatory related signaling pathways. J Funct Foods (2020) 64:103641. doi: 10.1016/j.jff.2019.103641

71. Liang Z, Yuan Z, Li G, Fu F, Shan Y. Hypolipidemic, antioxidant, and antiapoptotic effects of polysaccharides extracted from Reishi Mushroom, Ganoderma lucidum (Leysser: Fr) Karst, in mice fed a high-fat diet. $J$ Med Food (2018) 21(12):1218-27. doi: 10.1089/jmf.2018.4182

72. Parlee SD, Lentz SI, Mori H, MacDougald OA. Quantifying size and number of adipocytes in adipose tissue. Methods Enzymol (2014) 537:93-122. doi: 10.1016/B978-0-12-411619-1.00006-9

73. Livak KJ, Schmittgen TD. Analysis of relative gene expression data using real-time quantitative PCR and the $2-\Delta \Delta C T$ method. methods (2001) 25 (4):402-8. doi: 10.1006/meth.2001.1262 
74. Ding Y, Song Z, Li H, Chang L, Pan T, Gu X, et al. Honokiol Ameliorates High-Fat-Diet-Induced Obesity of Different Sexes of Mice by Modulating the Composition of the Gut Microbiota. Front Immunol (2019) 10:2800. doi: 10.3389/fimmu.2019.02800

75. Rosen ED, MacDougald OA. Adipocyte differentiation from the inside out. Nat Rev Mol Cell Biol (2006) 7(12):885-96. doi: 10.1038/nrm2066

76. Rosen ED, Walkey CJ, Puigserver P, Spiegelman BM. Transcriptional regulation of adipogenesis. Genes Dev (2000) 14(11):1293-307. doi: $10.1101 / \mathrm{gad} .14 .11 .1293$

77. Shen L, Li Q, Wang J, Zhao Y, Niu L, Bai L, et al. Mir-144-3p promotes adipogenesis through releasing c/ebp $\alpha$ from klf3 and ctbp2. Front Genet (2018) 9:677. doi: 10.3389/fgene.2018.00677

78. Rosen ED, Hsu C-H, Wang X, Sakai S, Freeman MW, Gonzalez FJ, et al. C/ $\mathrm{EBP} \alpha$ induces adipogenesis through PPAR $\gamma$ : a unified pathway. Genes Dev (2002) 16(1):22-6. doi: 10.1101/gad.948702

79. Um MY, Moon MK, Ahn J, Ha TY. Coumarin attenuates hepatic steatosis by down-regulating lipogenic gene expression in mice fed a high-fat diet. $\mathrm{Br} \mathrm{J}$ Nutr (2013) 109(9):1590-7. doi: 10.1017/S0007114512005260

80. Cui B, Liu S, Lin X, Wang J, Li S, Wang Q, et al. Effects of Lycium barbarum aqueous and ethanol extracts on high-fat-diet induced oxidative stress in rat liver tissue. Molecules (2011) 16(11):9116-28. doi: 10.3390/molecules 16119116

81. Kim J, Lee H, Lim J, Oh J, Shin SS, Yoon M. The angiogenesis inhibitor ALSL1023 from lemon-balm leaves attenuates high-fat diet-induced nonalcoholic fatty liver disease through regulating the visceral adiposetissue function. Int J Mol Sci (2017) 18(4):846. doi: 10.3390/ijms18040846

82. Kahn SE, Hull RL, Utzschneider KM. Mechanisms linking obesity to insulin resistance and type 2 diabetes. Nature (2006) 444(7121):840-6. doi: 10.1038/ nature 05482

83. Alsaggar M, Bdour S, Ababneh Q, El-Elimat T, Qinna N, Alzoubi KH. Silibinin attenuates adipose tissue inflammation and reverses obesity and its complications in diet-induced obesity model in mice. BMC Pharmacol Toxicol (2020) 21(1):8. doi: 10.1186/s40360-020-0385-8

84. Qatanani M, Lazar MA. Mechanisms of obesity-associated insulin resistance: many choices on the menu. Genes Dev (2007) 21(12):1443-55. doi: 10.1101/ gad.1550907

85. Fujisaka S, Usui I, Bukhari A, Ikutani M, Oya T, Kanatani Y, et al. Regulatory mechanisms for adipose tissue M1 and M2 macrophages in diet-induced obese mice. Diabetes (2009) 58(11):2574-82. doi: 10.2337/db08-1475

86. Chawla A, Nguyen KD, Goh YS. Macrophage-mediated inflammation in metabolic disease. Nat Rev Immunol (2011) 11(11):738-49. doi: 10.1038/ nri3071

87. Ley RE, Turnbaugh PJ, Klein S, Gordon JI. Human gut microbes associated with obesity. nature (2006) 444(7122):1022-3. doi: 10.1038/4441022a

88. Liu W, Zhao S, Wang J, Shi J, Sun Y, Wang W, et al. Grape seed proanthocyanidin extract ameliorates inflammation and adiposity by modulating gut microbiota in high-fat diet mice. Mol Nutr Food Res (2017) 61(9):1601082. doi: 10.1002/mnfr.201601082

89. Gil-Cardoso K, Ginés I, Pinent M, Ardévol A, Blay M, Terra X. Effects of flavonoids on intestinal inflammation, barrier integrity and changes in gut microbiota during diet-induced obesity. Nutr Res Rev (2016) 29(2):234-48. doi: 10.1017/S0954422416000159

90. Amoozadeh Y, Dan Q, Xiao J, Waheed F, Szászi K. Tumor necrosis factor- $\alpha$ induces a biphasic change in claudin-2 expression in tubular epithelial cells: role in barrier functions. Am J Physiol Cell Physiol (2015) 309(1):C38-50. doi: 10.1152/ajpcell.00388.2014

91. Monteiro R, Azevedo I. Chronic inflammation in obesity and the metabolic syndrome. Mediators Inflammation (2010) 2010:10. doi: 10.1155/2010/289645

92. Luck H, Tsai S, Chung J, Clemente-Casares X, Ghazarian M, Revelo XS, et al. Regulation of obesity-related insulin resistance with gut anti-inflammatory agents. Cell Metab (2015) 21(4):527-42. doi: 10.1016/j.cmet.2015.03.001

93. Kolsi RBA, Jardak N, Hajkacem F, Chaaben R, El Feki A, Rebai T, et al. Antiobesity effect and protection of liver-kidney functions by Codium fragile sulphated polysaccharide on high fat diet induced obese rats. Int J Biol Macromolecules (2017) 102:119-29. doi: 10.1016/j.ijbiomac.2017.04.017

94. Aida F, Shuhaimi M, Yazid M, Maaruf A. Mushroom as a potential source of prebiotics: a review. Trends Food Sci Technol (2009) 20(11-12):567-75. doi: 10.1016/j.tifs.2009.07.007
95. Xu X, Zhang X. Lentinula edodes-derived polysaccharide alters the spatial structure of gut microbiota in mice. PloS One (2015) 10(1):e0115037. doi: 10.1371/journal.pone.0115037

96. Jayachandran M, Xiao J, Xu B. A critical review on health promoting benefits of edible mushrooms through gut microbiota. Int J Mol Sci (2017) 18 (9):1934. doi: 10.3390/ijms18091934

97. Brownlee I, Fairclough A, Hall A, Paxman J. The potential health benefits of seaweed and seaweed extract. (2012).

98. Michida H, Tamalampudi S, Pandiella SS, Webb C, Fukuda H, Kondo A. Effect of cereal extracts and cereal fiber on viability of Lactobacillus plantarum under gastrointestinal tract conditions. Biochem Eng J (2006) 28(1):73-8. doi: 10.1016/j.bej.2005.09.004

99. Kaefer CM, Milner JA. The role of herbs and spices in cancer prevention. J Nutr Biochem (2008) 19(6):347-61. doi: 10.1016/j.jnutbio.2007.11.003

100. Deng C, Shang J, Fu H, Chen J, Liu H, Chen J. Mechanism of the immunostimulatory activity by a polysaccharide from Dictyophora indusiata. Int J Biol Macromolecules (2016) 91:752-9. doi: 10.1016/ j.ijbiomac.2016.06.024

101. Wang W, Song X, Gao Z, Zhao H, Wang X, Liu M, et al. Antihyperlipidemic, antioxidant and organic protection effects of acidicextractable polysaccharides from Dictyophora indusiata. Int J Biol Macromolecules (2019) 129:281-92. doi: 10.1016/j.ijbiomac.2019.01.182

102. Wang W, Song X, Zhang J, Li H, Liu M, Gao Z, et al. Antioxidation, hepaticand renal-protection of water-extractable polysaccharides by Dictyophora indusiata on obese mice. Int J Biol Macromolecules (2019) 134:290-301. doi: 10.1016/j.ijbiomac.2019.05.028

103. Van Herpen N, Schrauwen-Hinderling V. Lipid accumulation in nonadipose tissue and lipotoxicity. Physiol Behav (2008) 94(2):231-41. doi: 10.1016/j.physbeh.2007.11.049

104. Lu Y, Zhao A, Wu Y, Zhao Y, Yang X. Soybean soluble polysaccharides enhance bioavailability of genistein and its prevention against obesity and metabolic syndrome of mice with chronic high fat consumption. Food Funct (2019) 10(7):4153-65. doi: 10.1039/C8FO02379D

105. Sima C, Montero E, Nguyen D, Freire M, Norris P, Serhan CN, et al. ERV1 overexpression in myeloid cells protects against high fat diet induced obesity and glucose intolerance. Sci Rep (2017) 7(1):1-14. doi: 10.1038/s41598-01713185-7

106. Gustafson B, Hedjazifar S, Gogg S, Hammarstedt A, Smith U. Insulin resistance and impaired adipogenesis. Trends Endocrinol Metab (2015) 26 (4):193-200. doi: 10.1016/j.tem.2015.01.006

107. Gustafson B, Gogg S, Hedjazifar S, Jenndahl L, Hammarstedt A, Smith U. Inflammation and impaired adipogenesis in hypertrophic obesity in man. Am J Physiol Endocrinol Metab (2009) 297(5):E999-1003. doi: 10.1152/ ajpendo.00377.2009

108. Kim MH, Park S-J, Kim J-H, Seong JB, Kim K-M, Woo HA, et al Peroxiredoxin 5 regulates adipogenesis-attenuating oxidative stress in obese mouse models induced by a high-fat diet. Free Radical Biol Med (2018) 123:27-38. doi: 10.1016/j.freeradbiomed.2018.05.061

109. Juman S, Yasui N, Ikeda K, Ueda A, Sakanaka M, Negishi H, et al. Caffeic acid phenethyl ester suppresses the production of pro-inflammatory cytokines in hypertrophic adipocytes through lipopolysaccharidestimulated macrophages. Biol Pharm Bull (2012) 35(11):1941-6. doi: 10.1248/bpb.b12-00317

110. Karpe F, Dickmann JR, Frayn KN. Fatty acids, obesity, and insulin resistance: time for a reevaluation. Diabetes (2011) 60(10):2441-9. doi: 10.2337/db11-0425

111. Zhang H, DiBaise JK, Zuccolo A, Kudrna D, Braidotti M, Yu Y, et al. Human gut microbiota in obesity and after gastric bypass. Proc Natl Acad Sci (2009) 106(7):2365-70. doi: 10.1073/pnas.0812600106

112. Cani PD, Delzenne NM. Gut microflora as a target for energy and metabolic homeostasis. Curr Opin Clin Nutr Metab Care (2007) 10(6):729-34. doi: 10.1097/MCO.0b013e3282efdebb

113. Tilg H, Kaser A. Gut microbiome, obesity, and metabolic dysfunction. J Clin Investigat (2011) 121(6):2126-32. doi: 10.1172/JCI58109

114. Avila-Nava A, Noriega LG, Tovar AR, Granados O, Perez-Cruz C, PedrazaChaverri J, et al. Food combination based on a pre-hispanic Mexican diet decreases metabolic and cognitive abnormalities and gut microbiota dysbiosis caused by a sucrose-enriched high-fat diet in rats. Mol Nutr Food Res (2017) 61(1):1501023. doi: 10.1002/mnfr.201501023 
115. Zhang C, Zhang M, Wang S, Han R, Cao Y, Hua W, et al. Interactions between gut microbiota, host genetics and diet relevant to development of metabolic syndromes in mice. ISME J (2010) 4(2):232-41. doi: 10.1038/ismej.2009.112

116. Shen J, Obin MS, Zhao L. The gut microbiota, obesity and insulin resistance. Mol Aspects Med (2013) 34(1):39-58. doi: 10.1016/j.mam.2012.11.001

117. Ahmadi S, Nagpal R, Wang S, Gagliano J, Kitzman DW, Soleimanian-Zad S, et al. Prebiotics from acorn and sago prevent high-fat-diet-induced insulin resistance via microbiome-gut-brain axis modulation. J Nutr Biochem (2019) 67:1-13. doi: 10.1016/j.jnutbio.2019.01.011

118. Ferrario C, Statello R, Carnevali L, Mancabelli L, Milani C, Mangifesta M, et al. How to feed the mammalian gut microbiota: bacterial and metabolic modulation by dietary fibers. Front Microbiol (2017) 8:1749. doi: 10.3389/ fmicb.2017.01749

119. Wilson B, Whelan K. Prebiotic inulin-type fructans and galacto-oligosaccharides: definition, specificity, function, and application in gastrointestinal disorders. J Gastroenterol Hepatol (2017) 32:64-8. doi: 10.1111/jgh.13700

120. Le Chatelier E, Nielsen T, Qin J, Prifti E, Hildebrand F, Falony G, et al. Richness of human gut microbiome correlates with metabolic markers. Nature (2013) 500(7464):541-6. doi: 10.1038/nature12506
121. Lee H-G, Lu YA, Li X, Hyun J-M, Kim H-S, Lee JJ, et al. Anti-Obesity Effects of Grateloupia elliptica, a Red Seaweed, in Mice with High-Fat Diet-Induced Obesity via Suppression of Adipogenic Factors in White Adipose Tissue and Increased Thermogenic Factors in Brown Adipose Tissue. Nutrients (2020) 12(2):308. doi: 10.3390/nu12020308

122. An Y, Li Y, Wang X, Chen Z, Xu H, Wu L, et al. Cordycepin reduces weight through regulating gut microbiota in high-fat diet-induced obese rats. Lipids Health Dis (2018) 17(1):1-10. doi: 10.1186/s12944-018-0910-6

Conflict of Interest: The authors declare that the research was conducted in the absence of any commercial or financial relationships that could be construed as a potential conflict of interest.

Copyright (C) 2020 Kanwal, Aliya and Xin. This is an open-access article distributed under the terms of the Creative Commons Attribution License (CC BY). The use, distribution or reproduction in other forums is permitted, provided the original author(s) and the copyright owner(s) are credited and that the original publication in this journal is cited, in accordance with accepted academic practice. No use, distribution or reproduction is permitted which does not comply with these terms. 